\title{
Liquid flow focused by a gas: Jetting, dripping, and recirculation
}

\author{
Miguel A. Herrada, Alfonso M. Gañán-Calvo, Antonio Ojeda-Monge, Benjamin Bluth, and Pascual Riesco-Chueca \\ ESI, Universidad de Sevilla, Camino de los Descubrimientos, s/n 41092 Sevilla, Spain
}

(Received 8 December 2007; revised manuscript received 14 May 2008; published 25 September 2008)

\begin{abstract}
The liquid cone-jet mode can be produced upon stimulation by a coflowing gas sheath. Most applications deal with the jet breakup, leading to either of two droplet generation regimes: Jetting and dripping. The cone-jet flow pattern is explored by direct axisymmetric volume of fluid (VOF) numerical simulation; its evolution is studied as the liquid flow rate is increased around the jetting-dripping transition. As observed in other focused flows such as electrospraying cones upon steady thread emission, the flow displays a strong recirculating pattern within the conical meniscus; it is shown to play a role on the stability of the system, being a precursor to the onset of dripping. Close to the minimum liquid flow rate for steady jetting, the recirculation cell penetrates into the feed tube. Both the jet diameter and the size of the cell are accurately estimated by a simple theoretical model. In addition, the transition from jetting to dripping is numerically analyzed in detail in some illustrative cases, and compared, to good agreement, with a set of experiments.
\end{abstract}

DOI: 10.1103/PhysRevE.78.036323

PACS number(s): 47.55.D-, 47.20.Dr, 47.55.db

\section{INTRODUCTION}

The controllable production of small flowing geometries is a crucial challenge for chemical engineering [1] and bioindustry $[2,3]$. Drops, bubbles, jets, and recirculation cells in the microscale provide a useful platform for diverse technical applications. Here we concentrate on cone-jet flow patterns, looking at the streamline geometry in the jetting to dripping transition. Recirculating flow is shown to take place just before the transition. This may have been disregarded in some experimental setups, whose prime concern was the study of the cone and jet geometry, or the analysis of drop generation. Its study requires either specific flow visualization techniques or numerical simulation methods. The conditions for recirculation are extremely interesting, both as an indicator phenomenon associated to the jetting-dripping threshold, and as an attractive technological feature.

Small droplet generation by means of coflowing immiscible fluid streams has become widespread. The intrinsic smallness of the output droplets generally leads to small Reynolds number flows. Thus, a number of classic studies back to Taylor [4] including the recently blooming field of coflowing microfluidics take the low Reynolds number assumption for granted. For example, a simple scheme (a straight tube surrounds a coaxial, more slender tube, two immiscible fluids being fed through each tube) has been thoroughly explored by Suryo and Basaran [5] using a computer simulation: A locally extensional flow spontaneously develops at the tip of the elongated drop drawn by the coflowing liquid, causing the seemingly continuous ejection of extremely small droplets by tip streaming under certain parametrical combinations. This is obtained without the burden of complex geometry. It is hoped, therefore, that low Reynolds number coflowing small-droplet generation may become a hydrodynamic standard in a near future. Different setups have been proposed where the flow is driven by an external straining flow. Among them, the elegant analytical solution by Zhang [6] points to parametrical combinations where extremely thin fluid jets, even down to the molecular scale, could be continuously reached. Those jets, if con- firmed, would yield unimaginably small droplets upon breakup.

An interesting research field is concerned with the behavior of electrified cones and drops of leaky dielectric fluids. The problem of freely suspended liquid droplets deforming due to an applied electrostatic field was examined by Haywood, Renksizbulut, and Raithby [7]. Collins et al. [8] reported simulations and experiments supplying a comprehensive picture of the mechanisms of cone formation, jet emission and breakup that occur during electrohydrodynamic (EHD) tip streaming from a liquid film of finite conductivity. Lac and Homsy used a boundary integral method to describe the axisymmetric deformation and stability of a viscous drop in a steady electric field [9]. In the present paper, however, only nonelectrified fluids will be considered.

In spite of their generality and tractability, low Reynolds number flows are constrained by the requirement that the overall flow velocity does not grow above a certain threshold to ensure that inertial forces remain negligible. This constraint limits the overall productivity of low-Re systems. Coflowing with inertia was successfully explored, aiming at a reduction of the issued bubble diameter, by Oguz and Prosperetti [10]. Subsequently, new perspectives were open by the emergence of moderate-high Reynolds number flow focusing [11] as a high-productivity alternative to low Reynolds number coflowing systems. Compared to other coflowing techniques, flow focusing (FF) stands today as a mature microfluidic standard yielding steady capillary jets or droplets whose size is well below the scale of the flow boundaries. As originally conceived, FF aimed at the generation [11] of continuous steady microjets upon focusing by a coflowing gas stream at moderate-high Reynolds number. Furthermore, FF was shown [12] to produce perfectly monodisperse microbubble streams when the coflowing current is a liquid. A slight variation of the concept was subsequently introduced by Takeuchi et al. [13] to produce microbubbles. When the axisymmetric geometry originally proposed was reduced to a planar topology [14], particularly suitable for microfluidics, the scientific literature on flow focusing underwent an enormous boost [15-17]. In addition, axisymmetric multiple-phase FF leading to compound coaxial microjets 


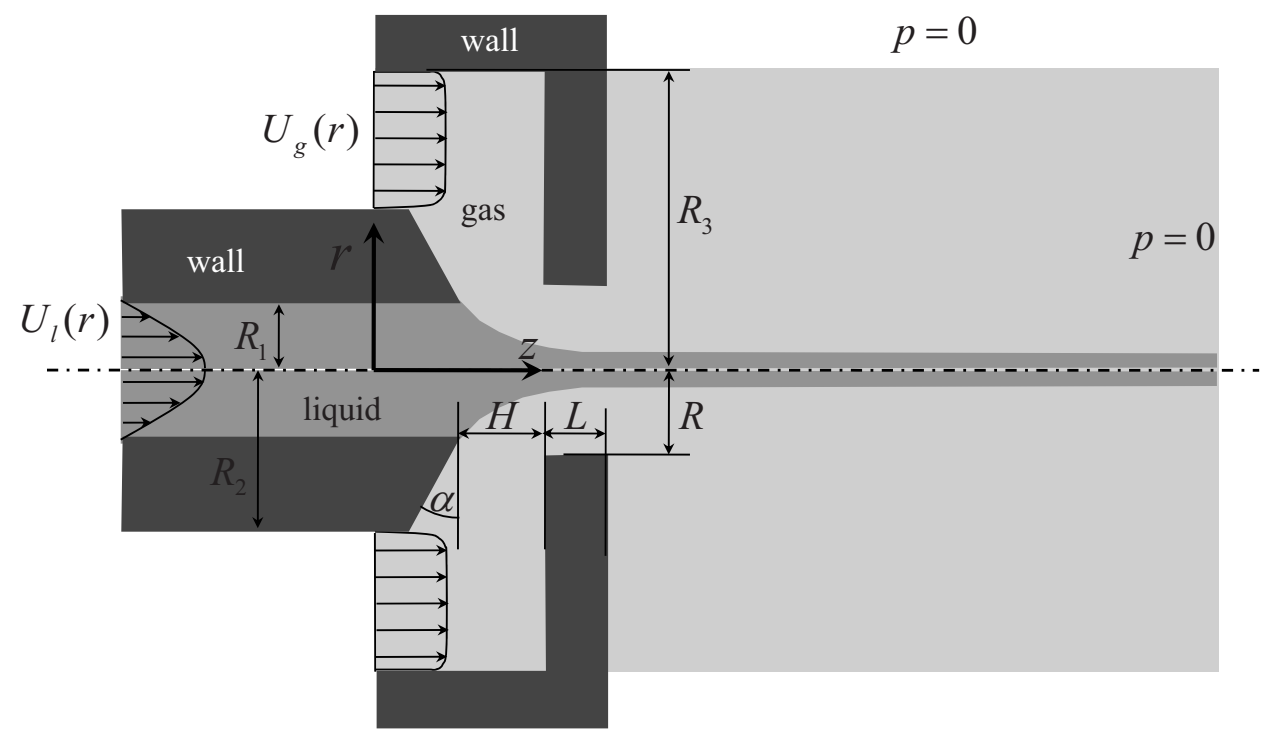

FIG. 1. Simulated boundary geometry and fluid flow domains.
[11] has been developed by other authors to produce microcapsules in a microfluidic setup at relatively low [18] and moderate Reynolds number [19].

The technological applications of FF were evident from its very inception. A crucial advance resulted from the combination of massive production (high production rates of microscopic fluidic entities) and accurate tailoring. Depending on the geometry and arrangement of the involved fluids (a decision determining which interfaces are to be created), the nature of the fluids involved, gas or liquid, and the system geometry, an output including nearly monodisperse microdroplets, bubbles or complex capsules can be obtained at an unprecedentedly controllable rate. Surface tension becomes a paramount ally in the conformation of discrete (generally spherical) fluidic units. Capillary jets have long ago been observed to give rise to continuous drop streams at fast emission rate upon Rayleigh axisymmetric breakup. Here, although surface tension is negligible compared to other driving forces in the global scale, it becomes the main driving agent for jet instability and breakup. Obtaining a jet is therefore the precondition for the creation of a fluid domain with higher velocities and smaller dimensions at no cost in terms of control; and surface tension is free to perform its conformation task in this new scale. Thus, as first proposed in FF, the steady capillary thin jet conformed by pressure forcing by an immiscible coflowing fluid provides favorable local conditions, a suitable environment for the generation of bubbles, capsules or droplets.

A FF capillary jet is driven by three main agents: fluid inertia, viscosity and surface tension. Owing to the simplicity of the slender jet geometry, which asymptotically renders all forces strictly additive in one dimension, FF can be scaled with the help of two dimensionless parameters: (i) the inertia to surface tension forces ratio (Weber number) and (ii) the viscous to surface tension ratio (capillary number). Other classic numbers such as Reynolds are combinations of the former. Nevertheless, as early noticed [11], an intrinsic feature of FF, namely the presence of a focusing fluid, gives rise to supplementary influences issuing from the correlation between the properties of the focusing and focused fluids. In particular, when a liquid is being focused by a gas, the gas sheath flows much faster than the liquid jet at the exit orifice (Fig. 1). Thus, in addition to the extensional viscous forces at the neck of the meniscus, transversal viscous diffusion of momentum causes a nontrivial axial velocity profile. Some simplifying assumptions have been adopted, yielding accurate first-order solutions [11,20,21]. However, they are not applicable to predict critical phenomena like the onset of steady jetting, or the jetting-dripping transition, as a function of the working parameters. These problems have been made analytically tractable at the expense of a drastic geometry simplification, i.e., assuming infinite jet slenderness [22-24]. Such simplified models are predictive in a variety of situations, but FF systems exhibit an intrinsically threedimensional meniscus from which the jet or the small droplets issue. Simultaneous modeling of the meniscus and the jet goes beyond the scope of present theoretical frameworks. Thus, numerical simulation or experiment are the only avenue to discern the physics of the fluid emission and its parametric conditions. Some further insight can be gained by general scaling laws. This is the approach chosen for the research presented here.

Many authors have applied numerical simulation [25] to this class of problems, where it has supplied welcome information on the droplet dynamics of complex flows [26]. A significant number of studies have been proposed on microfluidic FF devices; occasional comparison with experimental data is provided to validate the numerical model. A liquidliquid configuration for the production of microemulsions has been simulated [27] to good agreement with experiments [14]. Other authors have considered the microbubbling setup $[17,28]$, where good experimental fit is also obtained [15].

In this work, we make use of numerical simulation, with some experimental support, to study the generation of a liquid jet focused by gas in an axisymmetric FF device, at moderate-high Reynolds numbers. The jet diameters obtained in the simulation are in good agreement with our experiments and scaling laws [11], a fact that fully validates our hypotheses. Among other findings, we determine the flow rate at the jetting-dripping transition for two combina- 
tions of Reynolds and Weber numbers. We observe that either the jet or the cusplike meniscus are responsible for the global instability of the system, which drives it into welldefined dynamical cycles (global dripping). A detailed description of the flow pattern sheds light on the physics of the jetting-dripping transition and the peculiar appearance of these two regimes in coflow problems, as opposed to faucet jetting and dripping.

One of the key findings of the simulation is the occurrence, under favorable driving conditions, of a recirculation cell in the meniscus. This is in perfect analogy to recirculating meridian fluid flows observed inside Taylor cones when electrospraying liquids with sufficiently large values of both the viscosity and the electrical conductivity [29,30]. Additionally, in experiments aiming at the production of tip streaming patterns in liquid-liquid two-dimensional FF (surfactant treated interface), the streamline image of fluorescent particles seeding the flow of the internal, aqueous liquid during thread formation, was shown to consist of symmetric recirculation vortices [31]. In both types of motion, either driven by the electrical stresses acting at the cone surface or by the external focusing flow, the liquid flows towards the meniscus (cone) tip, along the generatrix, and away from it along the axis. The problem under consideration here is comparable to these other instances of recirculating cell, because the driving action of the gas sheath, which causes a strong tangential forcing at the interface, plays a similar role to either tangential electric stress [29,30], surfactant-aided liquid-liquid interaction [31], or purely surfactant-driven tipstreaming $[32,33]$. In this work, scaling arguments are developed to describe the size and occurrence of purely FF recirculation cells.

\section{GOVERNING EQUATIONS AND BOUNDARY CONDITIONS}

The axisymmetric flow-focusing device and the computational domain used are sketched in Fig. 1. A constant liquid flow rate $Q_{l}$, flowing through a capillary tube (outer diameter $D_{2}=2 R_{2}$, inner diameter $D_{1}=2 R_{1}$ ), is forced through a coaxial round orifice of diameter $D=2 R$ (nozzle) located at a downstream distance $H$ from the tube outlet. The liquid stream is drawn by a constant flow rate $Q_{g}$ of focusing gas stream discharging through the nozzle into a infinitely large chamber. The gas flow is assumed incompressible, in asymptotic consistency with the low pressure drop at the exit orifice, a condition prevailing when maximum droplet size monodispersity is required. Therefore, the incompressible, axisymmetric and unsteady Navier-Stokes equations in cylindrical $(z, r, \phi)$ coordinates are used to describe the time evolution of both fluids.

Figure 1 also shows the boundary conditions: (a) At the liquid inlet, $z=-z_{l}$, a Hagen-Poiseuille profile, $U_{l}(r)=V_{1}[1$ $\left.-\left(r / R_{1}\right)^{2}\right]$, is specified; (b) at the gas inlet, $z=0, R_{2}<r<R_{3}$, a uniform axial flow, $U_{g}(r)=V_{2}$, is assumed. This assumption is based on the following: The gas Reynolds number under the conditions explored is relatively high (of the order of, or above 100), while most flow-focusing devices have a gas inlet length $L_{g}$ which is not much bigger than the width
$\Delta R=R_{3}-R_{2}$, so that the relevant dimensionless number for boundary layer development, $\rho_{g} U_{g}(\Delta R)^{2} /\left(\mu_{g} L_{g}\right)$ is sufficiently above unity; (c) on all solid walls we assume no slip and no penetration $\mathbf{u}=\mathbf{0}$; (d) at the axis $r=0$ a symmetry condition is applied; (e) the outlet discharge chamber has been modeled as a rectangular box, $z=z_{\text {out }}$ and $r=r_{\text {out }}$ being two open surfaces where the pressure is set to zero. This assumption is discussed later on.

Note that the corresponding gas and liquid flow rates can be derived from the inlet velocity field,

$$
Q_{l}=\int_{0}^{R_{1}} 2 \pi r U_{l}(r) d r, \quad Q_{g}=\int_{R_{2}}^{R_{3}} 2 \pi r U_{g}(r) d r .
$$

Parametric studies of the dimensionless variables involved are carried out next. The velocity field $\mathbf{u}=(u, v)$ is scaled with the mean gas velocity at the nozzle $V$ $=Q_{g} /\left(\pi R^{2}\right)$, while length is scaled with the nozzle radius $R$, time $t$ with $R / V$, and pressure $p$ with $\rho_{g} V^{2}, \rho_{g}$ being the density of the focusing gas. All of the figures presented make use of dimensionless magnitudes based on this scaling. A single geometrical configuration is considered in this work, characterized by the following aspect ratios: $R_{1} / R=0.75$, $R_{2} / R=1.75, R_{3} / R=3.5, H / R=1$, and $L / R=0.75$. We have chosen a liquid-gas combination where

$$
\frac{\rho_{l}}{\rho_{g}}=833.33, \quad \frac{\mu_{l}}{\mu_{g}}=55.55,
$$

$\rho$ and $\mu$ being the density and viscosity of the liquid (subindex $l$ ) and the gas $(g)$. This choice is representative of the experimental jetting of air-focused water. The problem is governed in addition by the Reynolds and Weber numbers

$$
\begin{gathered}
\operatorname{Re}=\frac{\rho_{g} V R}{\mu_{g}}, \\
\mathrm{We}=\frac{\rho_{g} V^{2} R}{\sigma} ;
\end{gathered}
$$

$\sigma$ being the surface tension between the two phases. $Q$ is defined as the flow rate quotient,

$$
Q=Q_{l} / Q_{g} \text {. }
$$

For a given value of $\mathrm{Re}$ and We, we wish to analyze the formation of a steady liquid jet and the dependence of the flow on the quotient $Q$. In particular, we identify the minimum value of $Q, Q^{*}(\mathrm{Re}, \mathrm{We})$, below which the liquid jet ceases to be steady and a dripping regime is observed in the simulation. The regime is considered to be steady (and the jet convectively unstable) if the liquid meniscus remains steady for a sufficiently large period of time.

We should point out that in order to focus a jet of liquid by gas, moderate-high Reynolds numbers are needed. We consider in detail two different conditions for the focusing gas:

Case 1. $\mathrm{Re}=465.83, \mathrm{We}=8.137$.

Case 2. $\mathrm{Re}=931.666, \mathrm{We}=32.55$.

Each case will be explored under different flow rate quotients. 


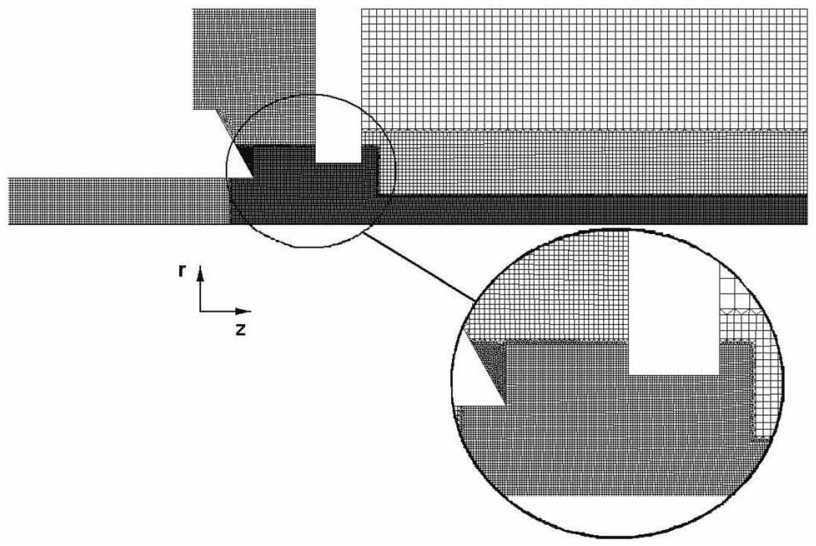

FIG. 2. Grid of the domain under study. A denser mesh is provided in areas where the interface is expected to lie. To avoid numerical diffusion of the interface, the interface region is defined with a higher density of nodes.

\section{NUMERICAL PROCEDURE}

In order to predict the interface geometry during the time evolution, several techniques have been used, falling into one of three categories. These are (i) interface tracking methods, including a moving mesh [34], (ii) front tracking and particle tracking schemes [35], and (iii) interface capturing methods, including volume of fluid (VOF) [36,37] and level set techniques [38]. We chose a VOF method consisting of two parts: an interface reconstruction algorithm to approximate the interface from the set of volume fractions and a VOF transport algorithm to determine the volume fraction at the new time level from the velocity field and the reconstructed front. The basic method is robust and flexible, and is based on widely used VOF schemes [39-42].

For convenience and with the aim of making our results readily reproducible for others, we have used the well-tested commercial solver FLUENT v 6.3 (laminar unsteady) to resolve the discretized mass continuity, momentum conservation, and the liquid volume fraction equations in the mesh depicted in Fig. 2, generated by commercial code GAMBIT in FLUENT $\mathrm{v}$ 6.2. The cells are polygons, mostly square and rectangular; some are triangles. Observe that the smallest cells lie between the needle edge and the nozzle, where the liquid meniscus is located, and in the near-axis region, where we expect the development of the liquid jet. The basic mesh should be sufficiently refined to capture, in the absence of the liquid, the strong velocity gradients experienced by the gas flow at the orifice region. In the grid shown in Fig. 2 the minimum cell radial and axial lengths are $(\Delta z)_{\min }=\left(\Delta_{r}\right)_{\min }$ $=0.02$. Several numerical tests with smaller size mesh cell have shown that this level of accuracy is comfortably sufficient to describe the gas flow pattern for the two cases considered $(\mathrm{Re}=465.83$ and 931.666). All results presented here were initially computed in that mesh. In all instances where $Q$ was very small, the results were recomputed in a refined mesh with rectangular cells in the nozzle and jet region, with $(\Delta z)_{\min }=(\Delta r)_{\min }=0.01$. Finally, only for the more difficult cases (case 2 with $Q$ small), the results were recomputed in a finer grid with $(\Delta z)_{\min }=(\Delta r)_{\min }=0.005$.
A factor requiring consideration is the location of the outlet boundaries. They are to be sufficiently remote from the nozzle to avoid the numerical reflection of pressure waves, since the pressure is artificially kept fixed at these boundaries during the time evolution of the flow. Moreover, the artificial boundary condition causes problems when the jet flows through the outflow boundary, and more acutely, when a string of drops flows through it. When a jet extends all the way to the outlet boundary of the flow domain, the pressure within the jet and the surrounding fluid cannot be equal owing to surface tension. However, for our particular problem, as will be shown later, these undesired effects are confined to a length below two diameters upstream of the boundary. Therefore, we have chosen simulations with a sufficiently large external chamber, $z_{\text {out }}=10$ and $r_{\text {out }}=3.5$, to minimize artificial boundary effects in the results obtained. On the other hand, the $z$ position where the inlet boundary conditions for the liquid are imposed, has been located sufficiently far away from the needle edge, at $z=z_{l}=-3$. This choice has been made, as will be shown later, because a liquid recirculation cell intrudes upon the capillary tube when $Q$ decreases. Therefore, in order to impose a Hagen-Poiseuille profile for the liquid velocity as a well-posed inlet boundary condition, this boundary should be set sufficiently far upstream from the recirculating region.

Tracking the interface between the phases is accomplished by solving a continuity equation for the volume fraction of one of the phases using an explicit time-marching scheme. The rest of the equations are solved implicitly. The time steps selected were fixed and sufficiently small to ensure that the global Courant number based on the mesh cell size, the mean velocity in the cell and the time step was always smaller than one. Regarding the spatial discretization of the equations, the third-order modified MUSCL scheme [43] is used to obtain the face fluxes whenever a cell is completely immersed in a single phase. When the cell is near the interface, the CICSAM algorithm is used [44]. The pressure corrections are computed with the body forces weighted scheme and the pressure-velocity coupling in segregated solver is treated with the PISO method [45]. All underrelaxation factors are set to one to avoid any numerical masking of fade-out effects in our physical problem.

\section{NUMERICAL RESULTS: DISCUSSION}

A fruitful interpretation of the results obtained needs to be situated in the frame of the literature on the dripping faucet. Shaw [46] gave rise to a rich and insightful series of studies, among them major contributions by Fuchikami et al. [47], Ambravaneswaran and co-workers [48-50], and Coullet, Mahadevan, and Riera [51]. To discriminate between the jetting and dripping modes, it is helpful to make use of the categories introduced by Ambravaneswaran and co-workers [49]:

(1) The dimensionless limiting length $L_{d} / R_{1}$ from the capillary edge to the extremity of the first drop at detachment.

(2) The ratio of the distance $L_{s}$ between the centers of mass of the drop that is about to form and the previously formed drop, and $L_{d}$. 
(3) The ratio of the volume of the drop that is about to form, $V_{d}$, to that of the drop that is attached from the capillary, $V_{p}$.

When undergoing the transition from dripping to jetting, the first parameter undergoes a sudden increase, while the two other ones experience an opposite trend. In general, the dripping mode is characterized by bulky drops, relatively distant from each other, whose diameter is considerably larger than the jet diameter from which they detach.

The usual categories applicable to faucet dripping need some adaptation before being used in a coflow problem, where there is considerable stretching of the cone-jet and droplet train by the coaxial current: The drops are deformed, their radial extent is limited, and they may undergo secondary breakup (particularly so in the dripping regime, whose bulky drops are more vulnerable to shear) after detachment from the filament. Therefore, under coflowing, the classical aspect of the jetting and dripping regimes is modified, and the transition between them is not sharp: Such features are confirmed by experiment, as explained below. This is the reason why the behavior of the meniscus can be used as a further indicator of the jetting mode. Dripping leads to a pulsating meniscus, each detached drop giving rise to recoil and oscillating; while, in jetting, the detachment of the drops does not cause any fluctuation of the meniscus and jet (see Fig. 19). In a full dripping regime, these pulses are perfectly regular (see Fig. 14); in most cases a slender unsteady liquid ligament detaches from the meniscus and breaks up into droplets of heterogeneous size [52]. However, at the onset of dripping (a situation which will be labeled "incipient dripping"), completely irregular fluctuations of the meniscus are observed [49].

Accordingly we begin by studying the formation of a steady (convectively unstable) liquid jet in the FF device. Initially, the capillary needle is filled with liquid up to $z=0$ while the rest of the domain is filled with gas. We start the simulation from rest $(u=v=0)$ in the whole domain except at the inlet sections, where velocity profiles are prescribed. Figures 3(a)-3(h) shows the formation of a steady liquid jet for case 1 and $Q=0.004$, going through the stages of interface entry, meniscus growth, and jet consolidation. The shape of the liquid-gas interface is computed in the figure as the isolevel of the liquid volume fraction $\alpha=0.999$, obtained with the VOF method. Given that the flow might be unsteady, we consider that the meniscus jet has reached a steady condition whenever two conditions hold:

(1) The angle between the liquid meniscus and the radial coordinate at the capillary needle, $\theta(t)$, has reached a constant value in time, $\theta(t)=\theta_{o}$.

(2) Both the jet diameter at the nozzle inlet, $d_{\text {in }}(t)$, and at the nozzle exit, $d_{\text {out }}(t)$, should reach a steady regime or a stable oscillating regime around a mean value; these quantities are of course to stay above zero. This amounts to excluding jet breakup in the nozzle region, a feature associated with a nonslender jet and possible dripping behavior (unsteady meniscus jet).

Here, $d_{\text {in }}(t)$ and $d_{\text {out }}(t)$ are computed at each time step, by integrating radially the liquid volume fraction, $\alpha$, at the nozzle inlet, $z=2$, and at the exit, $z=2.75$,

$$
d_{\text {in }}(t)=2 \sqrt{2 \int_{0}^{1} \alpha(t, z=2, r) r d r}
$$
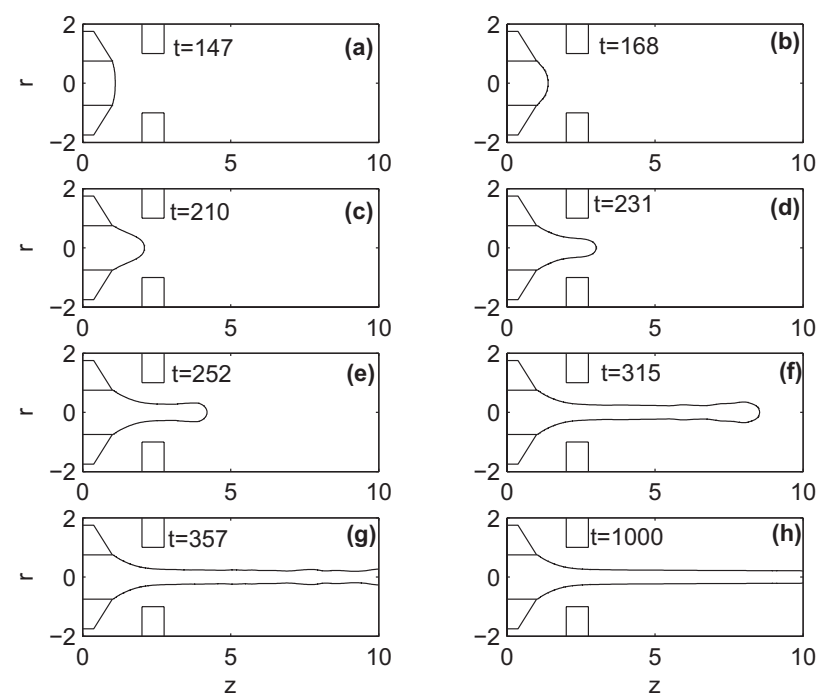

FIG. 3. A sequence of snapshots from the simulated growth of an eventually steady jet (case $1, Q=0.004$ ): (a) The interface arrives to the needle edge; (b) and (c) the meniscus grows in the nozzle region; (d)-(f) a jet begins to issue from the nozzle; (g) and (h) the meniscus-jet system is steady.

$$
d_{\text {out }}(t)=2 \sqrt{2 \int_{0}^{1} \alpha(t, z=2.75, r) r d r} .
$$

For sufficiently large $Q$, as illustrated in Fig. 3, both $d_{\text {in }}$ and $d_{\text {out }}$ evolve towards a steady value.

However, oscillations of these two quantities are observed when $Q$ is reduced. For example, Fig. 4 shows the time oscillation of $d_{\text {in }}$ and $d_{\text {out }}$ for case 1 and $Q=0.0006$ after allowing a steady jet to develop. It can be observed that the jet diameter at the nozzle exit is smaller than at the inlet; mass conservation arguments imply the inlet velocity to be smaller than the outlet velocity (in inverse proportion to the diameter squared). This explains why the oscillation frequencies of the jet diameter are shorter at the outlet. Although the oscillation of the jet in the nozzle region may play an important role in the dynamics of the droplets generated upon jet breakup, our main concern here is to characterize the jet diameter, the angle of the meniscus at the attachment, $\theta$, and the flow structure inside the meniscus as a function of $Q$. Since the flow is unsteady, we will use a mean value of the jet diameter at the nozzle inlet and outlet as defined by

$$
\bar{d}_{\text {in }}=\frac{1}{T} \int_{t_{i}}^{T+t_{i}} d_{\text {in }}(t) d t, \quad \bar{d}_{\text {out }}=\frac{1}{T} \int_{t_{i}}^{T+t_{i}} d_{\text {out }}(t) d t,
$$

where $t_{i}$ is a time position once a steady jet has developed and $T$ is a time period long enough to ensure a significative mean value. For example, selecting $t_{i}=0$ and $T=500$ leads to $\bar{d}_{\text {in }}=0.2456$ and $\bar{d}_{\text {out }}=0.1527$ (conditions as in Fig. 4).

The procedure is the same for the two cases under consideration. The simulation is started from rest with a value of $Q$ sufficiently high to obtain a steady jet. Then, $Q$ is reduced and the solution is monitored in time until a new steady jet is obtained. Figure 5 shows the stabilized liquid-gas interface for case 1 and different $Q$. It should be pointed out that $Q$ 


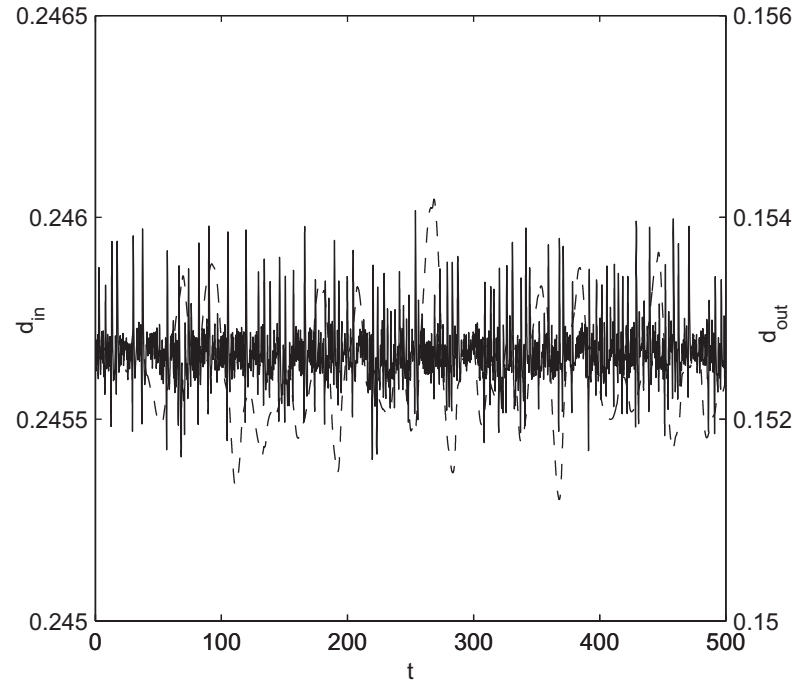

FIG. 4. Time dependence of the jet diameter, $d_{\text {in }}$ (dashed line, left-hand ordinates) and $d_{\text {out }}$ (continuous line, right-hand ordinates) for case 1 and $Q=0.0006$. Note (i) the smallness of the oscillation amplitudes and (ii) the higher frequency in the variation of $d_{\text {out }}$ as compared to $d_{\text {in }}$.

$=0.0004$ is the smallest flow rate compatible with a steady jet for case 1 . Therefore, it can be identified as the minimum flow rate $Q^{*}$ for steady jetting: $Q^{*}=0.0004$ for case 1 .

Figure 6 shows the interface for case 2 and different $Q$ values once a steady regime is reached. The smallest jetting flow rate here is $Q^{*}=0.0001$, four times smaller than in case 1. Accordingly, the smallest jet diameters are obtained for case 2. The jet diameter evolution is shown in Fig. 7, where the mean steady values $\bar{d}_{\text {in }}$ and $\bar{d}_{\text {in }}$ are plotted as a function of $Q$ for (a) case 1 and (b) case 2. To complete the picture, Fig. 8 shows the dependence of the meniscus angle $\theta$ with $Q$ for the two cases. In both examples, $\theta$ becomes smaller as $Q$ decreases (smaller flow rate quotient implies stronger focus-
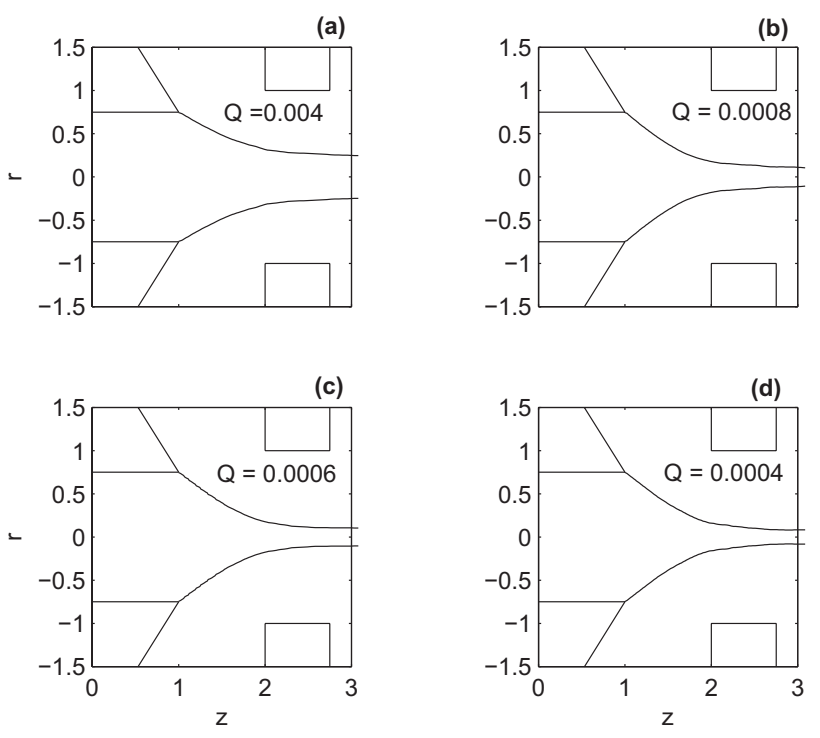

FIG. 5. Shape of the liquid-gas interface as a function of $Q$ (case $1)$.
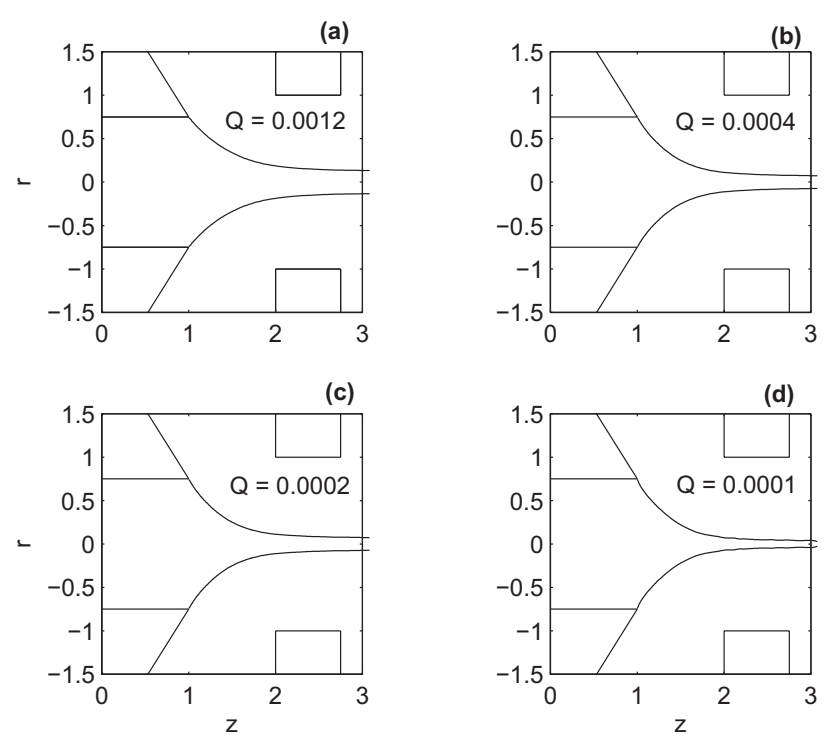
2).

FIG. 6. Shape of the liquid-gas interface as a function of $Q$ (case

ing action). Just before dripping, as the liquid flow rate is reduced, the angle appears to become independent from $Q$ : The interface geometry becomes invariant (local hydrostatic balance). The smallest angles are obtained in case 2 . This is to be expected since the normal pressure forces produced by the gas stream, which cause the focusing flow, are larger for that case.

Analyzing in more detail the structure of the flow inside the liquid meniscus in jetting mode, in the lower- $Q$ range, a meniscus recirculation cell is observed, in analogy with other coflowing systems [5,31] and Taylor cones [29]. Figure 9 shows instantaneous streamlines for case 1 and different values of $Q$. The recirculation increases when $Q$ decreases, the cell penetrating into the capillary needle. Figure 10 depicts instantaneous streamlines for case 2 and four different values of $Q$. Again, a recirculation region appears before the meniscus jet system ceases to be steady. The size of the recirculation can be calculated by finding the two $z$ positions where the velocity at the axis becomes zero.

Figure 11 shows the axial velocity at the axis, $u_{\mathrm{axis}}$, as a function of $z$ for five different values of $Q$ : (a) case 1 and (b) case 2. It is worth observing that $u_{\text {axis }}$ is roughly uniform inside the capillary needle, its value being given by the
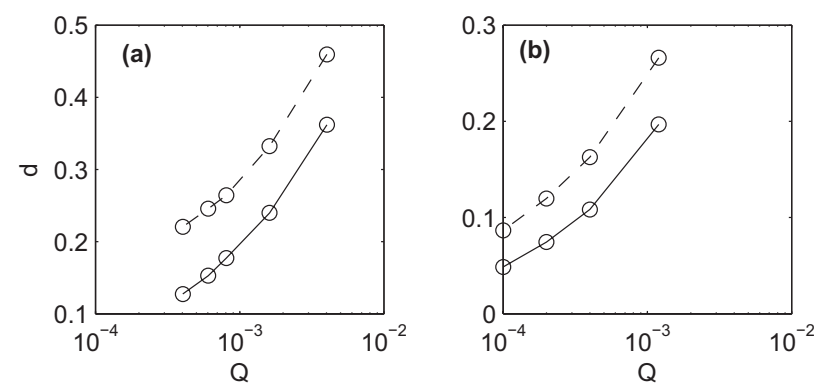

FIG. 7. Mean jet diameter at the inlet (dashed line) and at the exit (solid line) of the nozzle orifice versus the flow rate quotient $Q$. (a) Case 1, (b) case 2. 

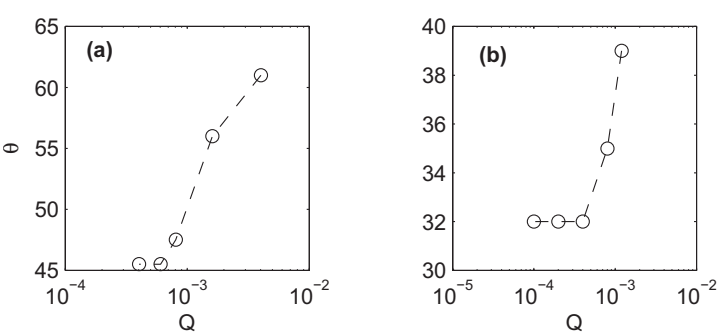

FIG. 8. Angle $\theta$ (degrees) versus flow rate quotient $Q$. (a) Case 1 , (b) case 2 .

Hagen-Poiseuille expression; in the nozzle region it increases owing to the focusing effect of the gas stream, which creates the issuing jet. A region where $u_{\text {axis }}$ decreases is located in the meniscus region, between the capillary and the nozzle; note that when $Q$ decreases, a local minimum of $u_{\text {axis }}$ is observed at a given position $z=z_{\min }$ in the meniscus region. If $Q$ is sufficiently small, $u_{\text {axis }}$ becomes negative near the local minimum in a region delimited by the two $z$ positions, $z_{1}$ and $z_{2}$, where $u_{\text {axis }}=0$. Therefore, the size of the recirculation region, $s_{R}$, observed in Figs. 10 and 11, can be computed as $s_{R}=z_{2}-z_{1}$. There is a threshold value of $Q, Q_{R}$, below which a recirculation pattern is observed. At the threshold flow rate $u_{\text {axis }}=0$ at $z_{1}=z_{\min }=z_{2}$ and $u_{\text {axis }}>0$ elsewhere.

Figure 12 shows $s_{R}$ as function of $Q$ for (a) case 1 and (b) case 2. Looking back at Fig. 9, note that the size of the recirculation cell increases as $Q$ decreases. In situations of incipient recirculation ( $Q$ smaller than but similar to $\left.Q_{R}\right)$ this growth appears to be linear, as derived later from dimensional arguments. In Fig. 12, the discrete points " $\bigcirc$ " have been obtained directly from the simulations. The dashed lines are linear interpolations computed in the recirculating regime, $s_{R}>0$. The linear interpolation is not only in good agreement with the data but also provides a reliable approximation to compute $Q_{R}$. The estimations are $Q_{R}=0.001453$ for case 1 and $Q_{R}=0.000708$ for case 2. According to the
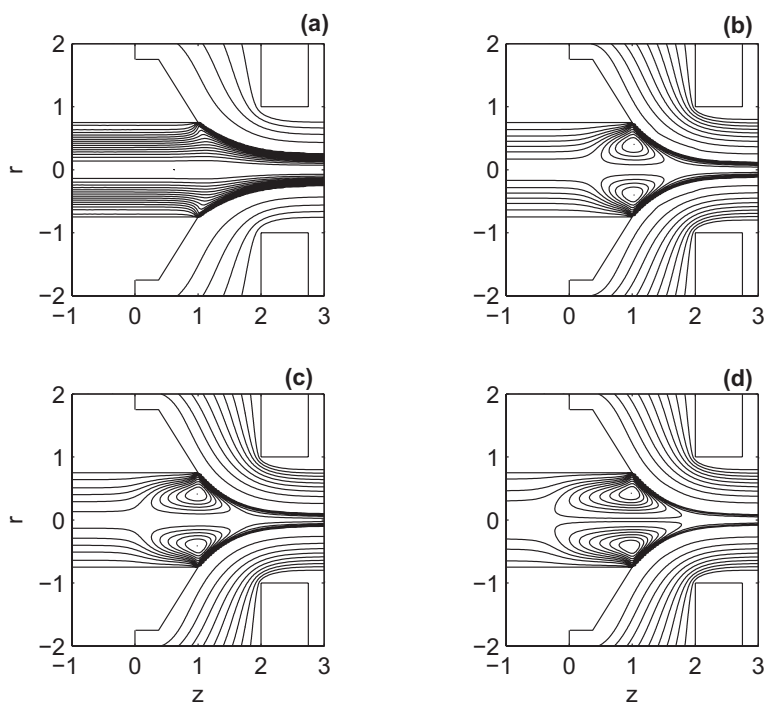

FIG. 9. Instantaneous streamlines at four different flow rates quotients $Q$ for case 1: (a) $Q=0.004$, (b) $Q=0.0008$, (c) $Q$ $=0.0006$, and (d) $Q=0.0004$.
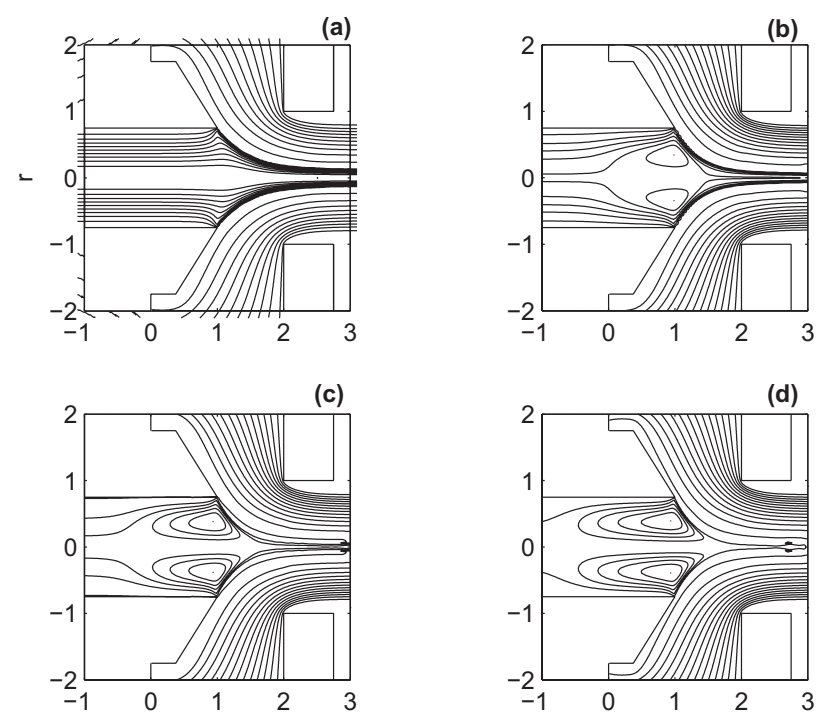

FIG. 10. Instantaneous streamlines with four different flow rate quotients $Q$ for case 2: (a) $Q=0.0012$, (b) $Q=0.0004$, (c) $Q$ $=0.0002$, and (d) $Q=0.0001$.

above, the linear expression relating the size of the recirculation region and the flow rate $Q$ is

$$
s_{R} \sim A\left(Q_{R}-Q\right)
$$

This means that $s_{R}$ is proportional to a back flow rate $Q_{b}$ made non-dimensional as $Q_{b} / Q_{g}=Q_{B}=\left(Q_{R}-Q\right)$ for a given set of fluid properties, geometry, and gas flow Reynolds number. The relative location of the jetting threshold $Q^{*}$ and the recirculation threshold $Q_{R}$ is, in both cases, $Q^{*}<Q_{R}$. Therefore, recirculation can be taken as a dripping precursor: As far as can be gathered from our simulation, it always precedes global instability of the meniscus-jet system.

Finally, some results are presented with flow rate values below the jetting threshold. For $Q<Q^{*}$, with $Q$ close to $Q^{*}$, our simulations show the flow to exhibit different behaviors in a sequence: A period where a thin jet breaks up in the nozzle region alternates with other periods where a thin jet breaks up downstream of the nozzle. The irregular time behavior of the flow for $Q<Q^{*}$, but $Q$ close to $Q^{*}$ (incipient dripping) can be observed in Fig. 13, where $d_{\text {in }}$ (a) and $d_{\text {out }}$ (b) are shown as a function of time for case 1 and $Q$ $=0.000322$. For $Q<Q^{*}$ but $Q$ sufficiently different from $Q^{*}$,
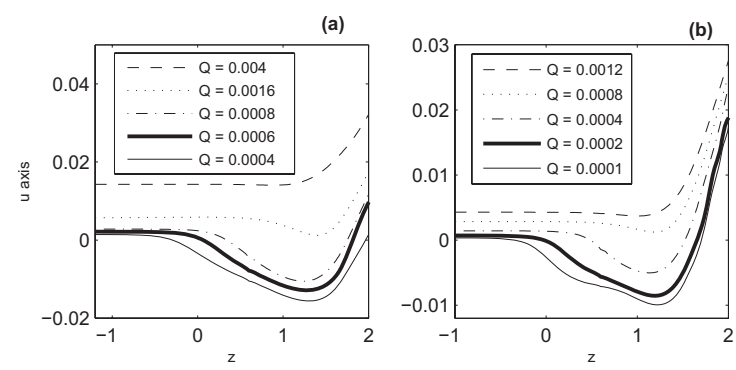

FIG. 11. Velocity at the axis versus $z$ for several flow rate quotients $Q$ : (a) Case 1 and (b) case 2 . 

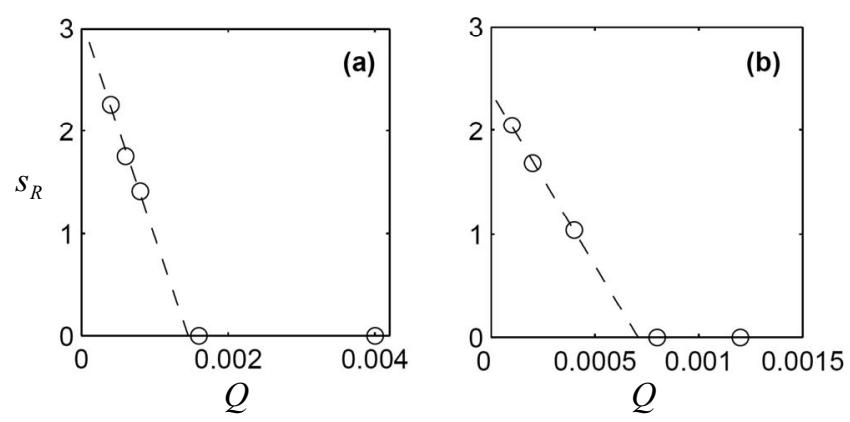

FIG. 12. Size of the recirculation $s_{r}$ as a function of $Q$ : (a) case 1 and (b) case 2.

the flow behavior becomes more regular and periodic with a unique dripping frequency.

As anticipated at the first part of this section, our results, while belonging to the dripping regime, are untypical in that the coflowing current gives rise to axial stretching of the jet and drops, so that unusual breakup geometries result. The radial extension of the drops is limited, and the axial stretching gives rise to secondary breakup, immediately after detachment. The pattern observed in Fig. 14 points to a dripping regime: It is perfectly periodic (transient jetting can therefore be excluded) and each period is associated with the filling up of a drop, its breakup from a thinning filament, and the recoil of this filament. Figures 14 shows a complete time sequence of a dripping process (case $1, Q=0.000241$ ). Figures 14(a)-14(e) show the growth of the meniscus and the formation of a jet issuing from the nozzle; Figs. 14(g) and 14(h) show the jet breakup into droplets of different sizes and the meniscus recoil. This is a periodic sequence, the period being $T \sim 210$ for each cycle. Figure 15 plots $d_{\text {in }}$ (a) and $d_{\text {out }}$ (b) as a function of time for this case. Initially, a liquid meniscus is growing with no jet production, and $d_{\text {in }}$ $=d_{\text {out }}=0$. Subsequently, a liquid jet issues and $d_{\text {in }}$ and $d_{\text {out }}$ become positive. Both diameters reach a maximum at a certain time and then start to decrease. Finally, the jet breaks into droplets, $d_{\text {in }}$ and $d_{\text {out }}$ are set to zero and the process begins anew. In spite of the observed differences, the dripping process in this case is quite similar to regular faucet dripping, the time period being mainly imposed by the filling of the meniscus until reaching a critical volume.

A similar situation is observed in case 2. Figure 16 shows a complete time sequence of a regular dripping process with $Q=0.000$ 04. Figures 16(a)-16(d) show the meniscus growth and the emission of a jet, much thicker and longer than ob-
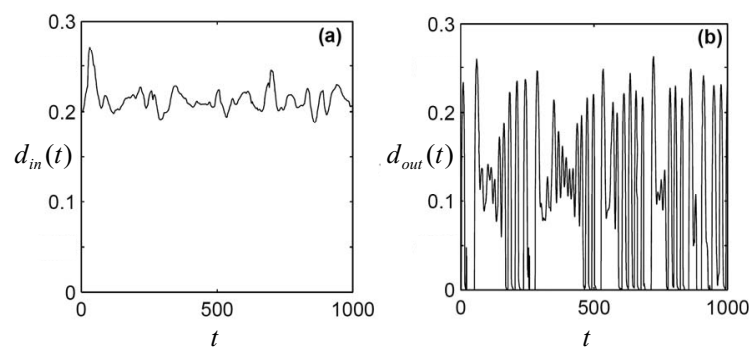

FIG. 13. Time evolution of (a) $d_{\text {in }}$ and (b) $d_{\text {out }}$ in a irregular dripping regime for case 1 and $Q=0.000322$.
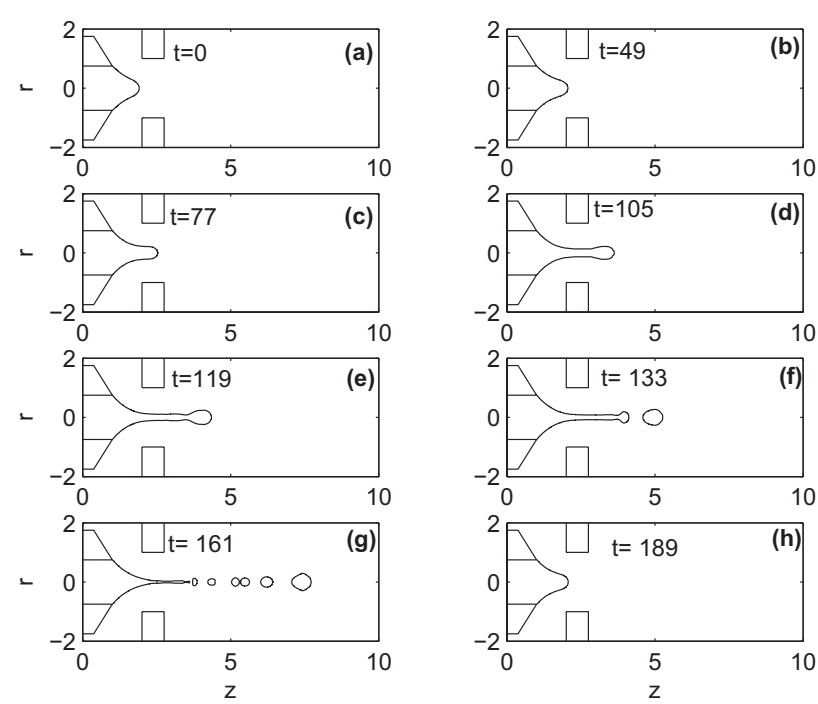

FIG. 14. A sequence of the dripping regime for case 1 and $Q$ $=0.000241$ involving a complete cycle.

served in Fig. 14. Again, this sequence is time periodic with a period $T \sim 500$ for each cycle [see $d_{\text {in }}$ (a) and $d_{\text {out }}$ (b) in Fig. 17 as a function of time].

Influence of the boundary conditions and the spatial and temporal resolution on the numerical results. The numerical problem addressed is quite complex: It involves a high speed stream of gas discharging through a nozzle into a infinitely large chamber plus a meniscus-liquid jet which may break into droplets within the finite numerical domain. This complexity leads to different time and spacial scales associated to a plurality of interacting physical phenomena (jet breakup due to capillary and Kelvin-Helmholtz instabilities, mixing layer instabilities in the main gas stream). Therefore, an accurate analysis of the jet breakup is difficult to achieve. On account of it, though our VOF method is fully reliable in qualitative terms as a predictor of jet breakup and drop formation, we have devoted this section to check that our numerical results (meniscus-jet shape as a function of $Q$ for different setups) are independent of the selected boundary conditions (BCs) and numerical meshes.

As indicated above, the most problematic simulation choice is setting $p=0$ at the outlet boundaries, since any jet or a drop crossing the boundary is influenced by the strong and artificial restriction that the pressure remains fixed. Our choice is a simplification $(p=0)$ which takes advantage of
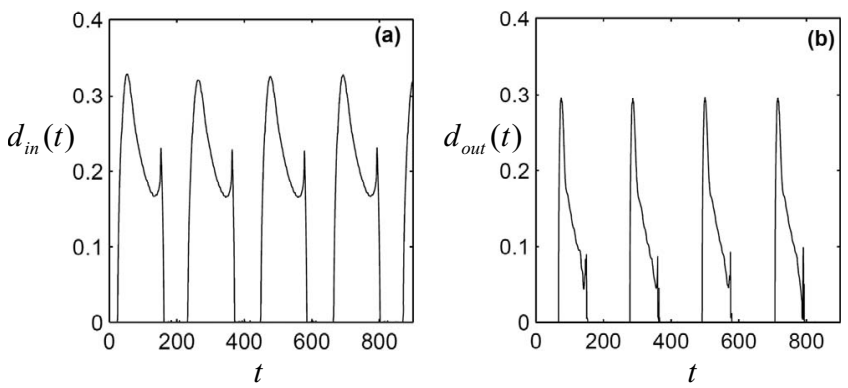

FIG. 15. Time evolution of (a) $d_{\text {in }}$ and (b) $d_{\text {out }}$ in a dripping regime for case 1 and $Q=0.000241$. 

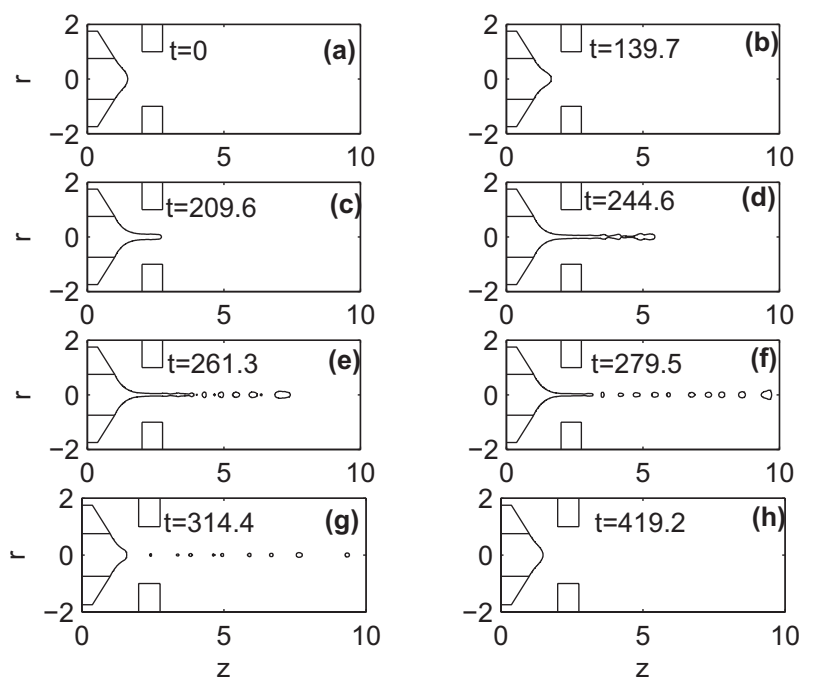

FIG. 16. A sequence of the dripping regime for case 2 and $Q$ $=0.00004$ involving a complete cycle.

the essentially parabolic character of the equations. A second option has been explored, the so-called outflow conditions (assuming uniformity, i.e., Neumann type), but they give rise to a false constraint on the flow pattern, because they imply that the gas flow is coaxial. A minisymposium held in 1994 on the open boundary condition problem in incompressible flow, by Sani and Gresho [53] led to the concluding remark: "We have made some attempts at shedding more light on the difficult and unresolved area of seeking good open boundary conditions (OBCs) for incompressible flow simulations. It has been an exercise in frustration and we are not thrilled with the results obtained."

There is an evident inaccuracy involved in our $p=0$ choice: The pressure jump associated to an interface will lead to high local pressure inside the liquid jet or droplets. However, this assumption can be reconciled with our aim, which is not a study of the breakup process and its transient geometries. We are addressing a wider scale: the cone-jet flow pattern, and the general drop generation regime. To show that the distortion caused by this artificial $\mathrm{BC}$ is local and does not modify the global behavior at the cone-jet region, some exploration as been carried out. It can be shown that setting the external boundary sufficiently far downstream from the nozzle region, at $z_{\text {out }}=10$, the meniscus jet is not affected by the boundary condition. To show this, we have considered the worst scenario: We choose large liquid
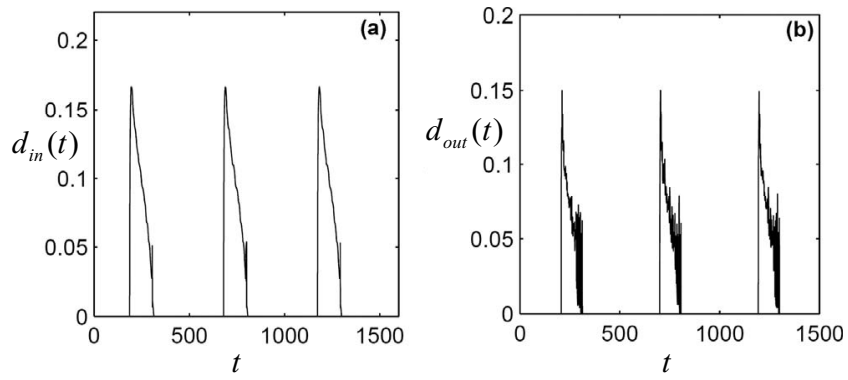

FIG. 17. Time evolution of (a) $d_{\text {in }}$ and (b) $d_{\text {out }}$ in a dripping regime for case 2 and $Q=0.00004$.
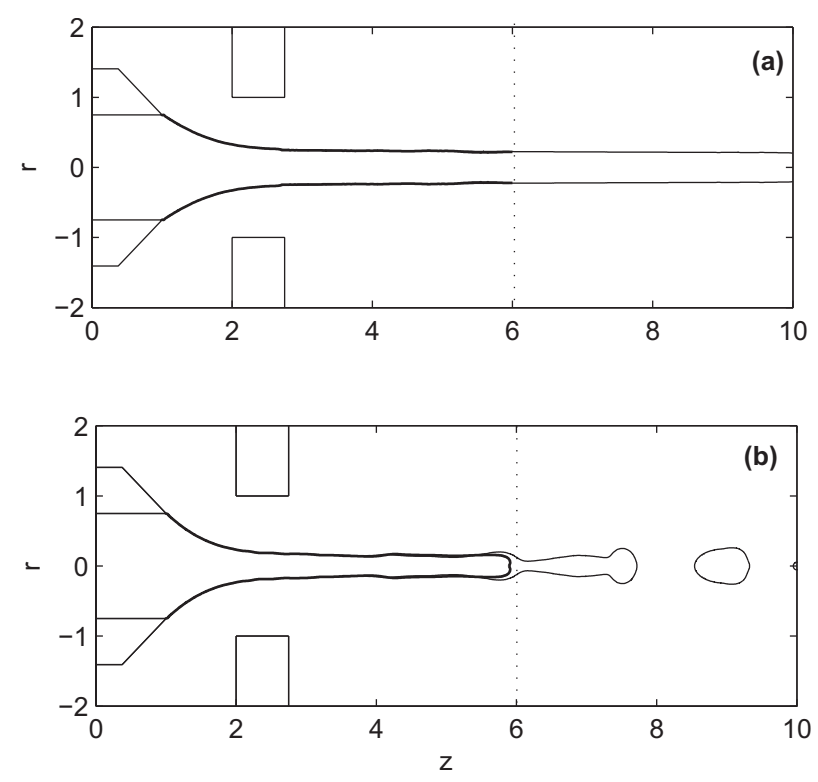

FIG. 18. The effect of the $p=0$ boundary condition on the liquid-gas interface comparing to downstream boundary locations, $z=6$ (thicker line) and $z=10$ (thinner line): (a) Data as in case 1 and $Q=0.004$; (b) data as in case 1 and $Q=0.0008$.

flow rates $Q$ and weak gas flow (case 1). Figure 18 shows the stabilized liquid-gas interface for case 1 and two different values of $Q$, computed in the original domain and in a shorter one. In Fig. 18(a), the jet does not break up within any of the two numerical domains and the jet and meniscus interface in the nozzle region is evidently not affected by the artificial $p=0$ boundary condition. The influence of the artificial BC is confined to a few diameters upstream of the downstream boundary. In the case considered in Fig. 18(b), the jet is breaking up into drops within the large domain. Even in this case, the meniscus and jet interface in the nozzle region is not affected by the artificial boundary condition.

Let us now show the consistency of the model by comparing the results in two different meshes. In this case again, we have considered the worst scenario, by selecting smaller values of the liquid flow rate ( $Q$ small) and a large gas flow (case 2), since thinner jets are obtained in these cases. Figure 19(a) shows a instantaneous picture of a steady (convectively unstable) liquid jet breaking up into drops (case 1, $Q$ $=0.0001$ ) computed in two different meshes. Observe that although we are comparing the liquid interface at two different times and with different spatial resolution, the shape of the meniscus of the liquid in the nozzle zone coincides. The main difference is that the liquid jet is slightly longer in the finer mesh. As mentioned, an accurate description of the jet breakup is not the objective of this paper. It requires specific analytic tools in order to capture the diverse physical phenomena involved.

This can be illustrated by Fig. 19(a), showing instantaneous contours of the vorticity field for this case, computed with the best spatial resolution. It can be seen that, owing to the large density and velocity difference between the liquid and the gas, the vorticity is large in the liquid-gas boundary layer which develops at the liquid-jet meniscus. When the jet breaks up, the behavior of the flow around the drops is simi- 

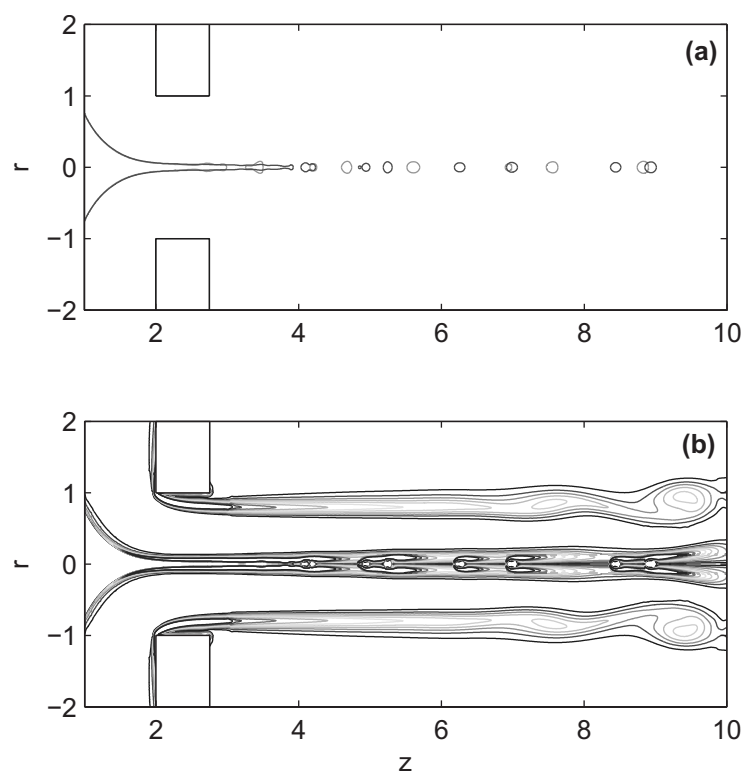

FIG. 19. The effect on the liquid-gas interface of the spatial resolution: (a) A general view of case $2, Q=0.0001$ where the grey line is the solution computed in a mesh where $\left(\Delta_{z}\right)_{\min }=\left(\Delta_{r}\right)_{\min }$ $=0.01$ and the black line is the solution obtained in a mesh with $\left(\Delta_{z}\right)_{\min }=\left(\Delta_{r}\right)_{\min }=0.005$; (b) contours of the vorticity field for the same case with the best spatial resolution.

lar to high Reynolds number flow around a round object (since the gas is traveling faster that the liquid drops). Therefore, boundary layer separation is to be expected at the surface of the drops, and a wake will originate downstream of the drop. In addition to this, a mixing layer is developing downstream of the nozzle edge owing to the difference between the velocity of the gas flowing through the nozzle and the stagnant atmosphere surrounding the outlet. This mixing layer yields to the development of vortices as shown in the figure. Figure 20(a) shows the time evolution of the vorticity magnitude at a point of this mixing layer computed with two different time steps. Strong fluctuations of the vorticity magnitude are apparent. The flow evolution shows some sensitivity to the resolution, particularly as the simulation time increases. However, both time resolutions are reliable to predict the characteristic frequencies of the problem at the observation point. Figure 20(b) show the frequency content of the vorticity as obtained by applying the fast Fourier transform (FFT) to the two signals. The main frequency $\omega_{1}$ $\sim 0.46$ is related to the passage of the vortices generated in the gas mixing layer. There is a secondary characteristic frequency, $\omega_{2} \sim 0.56$, associated to the interaction between the mixing layer and the vorticity wake of the drops. This interpretation was strengthened by recomputing the flow without the liquid jet: The frequency content of the vorticity signal at the same observation point only showed the main frequency peak $\omega_{1} \sim 0.46$.

\section{COMPARISON WITH ANALYTICAL MODELS AND SCALING LAWS}

The first predictive model for the jet diameter $d_{j}$ at the orifice exit [11] assumes that viscous and capillary effects are
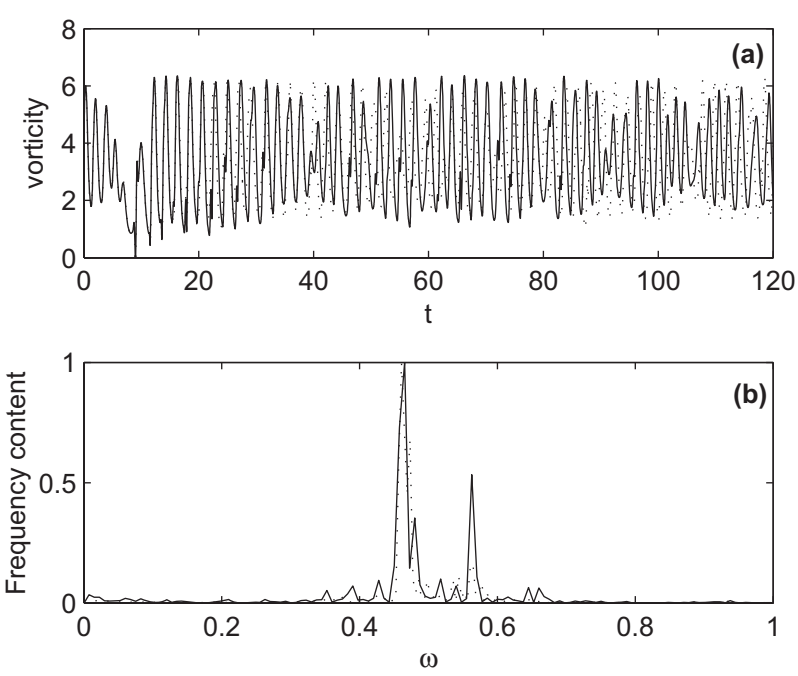

FIG. 20. (a) Comparison of the time evolution of the vorticity magnitude at the point $(z=9.5, r=0.995)$ for case 2 and $Q$ $=0.0001$ computed with $\Delta t=0.014$ (solid line) and with $\Delta t=0.028$ (dashed line); (b) shows the frequency content of the two signals.

small enough compared to liquid inertia. This demands large enough Reynolds and Weber numbers of the liquid jet, in reasonable agreement with most experimental conditions (common solvents including water, down to the micron scale). In this limit, the overall pressure difference $\Delta P$ $=\Delta P\left(Q_{g}\right)$ (pressure difference between the gas inlet and the gas outlet) imposed in the downstream direction (i.e., through the orifice), transmitted to the liquid stream by normal surface stresses, is converted into kinetic energy, so that

$$
\Delta P \simeq \frac{1}{2} \rho_{l} v^{2} \simeq \rho_{l} \frac{8 Q_{l}^{2}}{\pi^{2} d_{j}^{4}} \quad(\text { Ref. [11]) }
$$

which readily gives

$$
d_{j}=\left(\frac{8 \rho_{l}}{\pi^{2} \Delta P}\right)^{1 / 4} Q_{l}^{1 / 2} .
$$

Furthermore, the jet is assumed sufficiently small compared to the orifice diameter $D$ such that not only does it not touch the orifice borders, but also the boundary layers of the focusing fluid (gas) at the orifice and at the jet's surface are sufficiently small compared to the corona defined between the jet and the orifice. This is why $D$ does not enter expression (10). Neither does $D_{1}$ have any direct influence on the jet diameter; only as a parameter determining the liquid flow rate $Q_{l}$.

Interestingly, if viscous effects and surface tension are neglected, and we assume $d_{j} \ll D$, the only operating parameters left in the analysis are $\left\{\rho_{l}, \Delta P, Q_{l}\right\}$; using these three parameters, a scaling law identical to 10-regardless the constants-follows from dimensional reasoning. Figure 21 illustrates the accuracy of this first simple prediction. However, that expression does not provide information on how small the neglected effects are. Would it be possible to quantify both the dependence of the jet diameter on the three main parameters and the relative magnitude of each one of the neglected effects? The answer is yes, by retaining either 


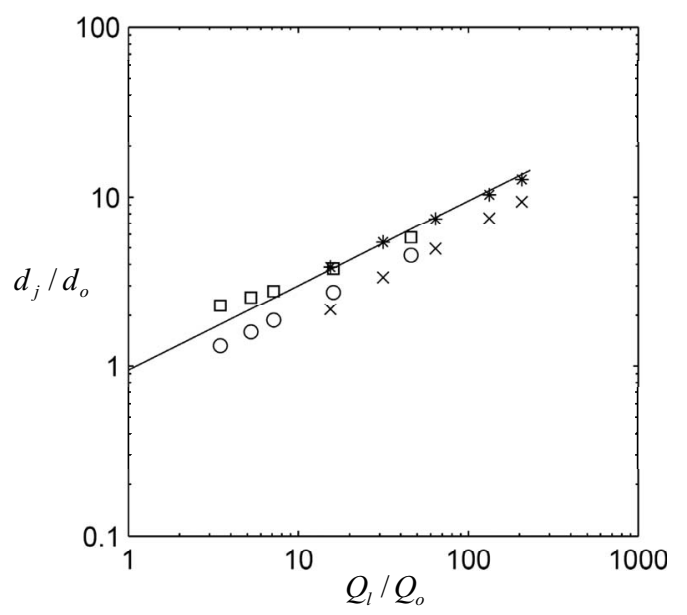

FIG. 21. Jet size measured at the entrance of the nozzle using $\bar{d}_{\text {in }}$ ( $\square$ correspond to case 1 and $*$ to case 2 ) and at the exit using $\bar{d}_{\text {out }}$ ( $\bigcirc$ correspond to case 1 and $\times$ to case 2 ) compared to the theoretical prediction (continuous line).

$\sigma, \mu_{l}$, or $D$ in the dimensional analysis, and using $\left\{\Delta P, \sigma, \rho_{l}\right\},\left\{\Delta P, \mu_{l}, \rho_{l}\right\}$, or $\left\{\Delta P, D, \rho_{l}\right\}$ as independent parameters, respectively.

In physical terms, the relative effect of surface tension may be determined by observing that the liquid Weber number must be of order unity $\left[\mathrm{We}_{l}=\rho_{l} 8 Q_{l}^{2} /\left(\pi^{2} \sigma d_{j}^{3}\right)\right]$ for a given pressure $\Delta P$, from which one obtains the limiting diameter $d_{o}$ and flow rate $Q_{o}$. The result is $d_{o}=\sigma / \Delta P$ and $Q_{o}$ $=\left(\sigma^{4} \rho_{l}^{-1} \Delta P^{-3}\right)^{1 / 2}$, so that dimensional analysis together with Eq. (10) readily yields

$$
d_{j} / d_{o}=k_{d}\left(Q_{l} / Q_{o}\right)^{1 / 2},
$$

where $k_{d}=\left(8 / \pi^{2}\right)^{1 / 4}$. This expression provides a first-order approximation to the jet diameter (asymptotically true for $\mathrm{Re} \rightarrow \infty)$ as far as $d_{j} \gg d_{o}$ (negligible surface tension). The ratio $Q_{l} / Q_{o}$ spans the whole domain from jetting to dripping-where $d_{j}$ becomes comparable to $d_{o}$. Jet diameters and flow rates comparable to $d_{o}$ and $Q_{o}$, respectively, lead to absolute instability, where the characteristic velocity of upstream capillary waves $O\left(\sigma \rho_{l}^{-1} d_{j}^{-1}\right)^{1 / 2}$ - a product of surface tension-becomes of the order of the downstream convective velocity, $Q_{l} / d_{j}^{2}$. Besides, Eq. (11) is explicitly independent of the orifice diameter $D$, an illustration of the jetting regime independently of its forcing geometry.

Similarly, viscous effects can be weighted by defining a viscosity-related length $d_{\mu}=\left(\mu_{l}^{2} \rho_{l}^{-1} \Delta P^{-1}\right)^{1 / 2}$ and flow rate $Q_{\mu}=\left(\mu_{l}^{4} \rho_{l}^{-3} \Delta P^{-1}\right)^{1 / 2}$. Using these and Eq. (10), an entirely analogous expression is obtained in the limit of dominant inertia, i.e., when $d_{j} \gg d_{\mu}$ and $Q_{l} \gg Q_{\mu}$,

$$
d_{j} / d_{\mu}=k_{d}\left(Q_{l} / Q_{\mu}\right)^{1 / 2} .
$$

This equation expresses the jet diameter as compared to a limit where viscous effects become important. Again, jet diameters and flow rates comparable to $d_{\mu}$ and $Q_{\mu}$, respectively, amount to non-negligible viscous effects and significant departures from predictions (11) or (12).
A third expression can be obtained in terms of the orifice diameter $D$, and the maximum liquid flow rate that can be ejected through the orifice for a given $\Delta P$ in the absence of viscous effects: $Q_{\max }=\left(\pi^{2} / 8\right)^{1 / 2} Q_{m}$, where-naturally- $Q_{m}$ $=\left(D^{4} \Delta P \rho_{l}^{-1}\right)^{1 / 2}$ is obtained from dimensional analysis using $\{\Delta P, D, \sigma\}$. Using (10) anew, one has

$$
d_{j} / D=\left(Q_{l} / Q_{\max }\right)^{1 / 2}=k_{d}\left(Q_{l} / Q_{m}\right)^{1 / 2} .
$$

This alternative expression reflects how close the experiment is from a situation where the entire orifice section is filled with liquid: It provides information-from continuity arguments - on the fraction of the orifice cross section occupied by the liquid jet.

Each of the above three expressions (11)-(13) amount to interesting but partial pictures of the particular working conditions of our system in a given flow situation. Taken as a whole, they provide a more complete picture on the FF jetting conditions. Some corrections can be obtained for several neglected effects [21].

\section{A. Correction for surface tension effects}

The liquid surface tension reduces the effective pressure drop $\Delta P_{l}$ in the liquid stream as

$$
\Delta P_{l}=\Delta P-2 \sigma / d_{j} .
$$

Consequently, the jet velocity decreases and its diameter increases accordingly. The resulting expression for the nondimensional jet diameter $d_{j} / d_{o}$, neglecting third-order terms proportional to $O\left(d_{o} / d_{j}\right) \ll 1$, reads as

$$
d_{j} / d_{o}=\left(8 / \pi^{2}\right)^{1 / 4}\left(Q_{l} / Q_{o}\right)^{1 / 2}+1 / 2 .
$$

In other words, the second-order correction of the jet diameter $d_{j}$ to account for surface tension effects is asymptotically equal to $d_{o} / 2$.

\section{B. Correction for liquid viscosity effects (extensional stresses)}

Assuming that the extensional viscous forces in the liquid are smaller than inertia, the balance of the different terms of the momentum equation, including the second-order terms of the expansion, leads to the following order of magnitude for the correction to the first-order diameter (10):

$$
d_{e}=O\left[d_{\mu}\left(\frac{Q_{\max }}{Q_{l}}\right)^{1 / 2}\right] .
$$

\section{Correction for tangential stresses owing to the gas stream}

In the same way, the diameter correction (decrease) owing to the momentum injected by the much faster gas stream through the jet surface is of the order of

$$
d_{g}=O\left(\frac{\mu_{g} U_{g} D}{\Delta P}\right)^{1 / 2},
$$

where $\mu_{g}$ and $U_{g}$ are the gas viscosity and velocity. The latter is of the order of $U_{g} \sim O\left(\Delta P / \rho_{g}\right)^{1 / 2}$, where $\rho_{g}$ is the gas density. 
The relative weight of these three corrections provides information on the importance of the surface tension and the viscosity of the liquid and gas phases. Interestingly, for most common solvents, these relative weights are of order unity. This happens to be the case when measuring the relative importance of the surface tension and the gas tangential stress effects for water focused by any gas at standard conditions. Therefore, since both corrections are opposite, the best agreement with experimentally measured jet diameters and numerical simulations is obtained, interestingly enough, using the first-order expression (10), or its alternative forms (11)-(13).

\section{Correction owing to the nozzle flow pattern}

The jet diameter as measured at the nozzle may also differ from the simplest theoretical prediction given by Eq. (10) because of local flow effects. A complex but symmetric structure develops owing to the coexistence of (i) a core potential flow and (ii) the detachment of a radially convergent boundary layer at the inner lip of the nozzle. In any real situation where the gas viscosity is nonzero and the continuum hypothesis holds, this flow pattern is not aptly described by the pure potential flow through a round orifice given by Morse and Feshback [54] (p. 1294) for a stationary discharge. The potential flow solution is characterized by an axial velocity distribution with a minimum value at the axis, $v(r=0)=2 Q_{g} /\left(\pi D^{2}\right)$ (one-half the average velocity through the orifice), and an infinite value at $r=D / 2, Q_{g}$ being the theoretical gas flow rate discharged. The actual flow geometry is characterized by the well-known vena contracta effect, a consequence of the radial momentum carried by the collapsing potential flow, which slips at the nozzle border owing to the boundary layer. The vena contracta flow exhibits an axial velocity distribution which echoes the potential flow solution, showing a local minimum velocity at the axis, and a maximum value at the streamlines coming just from the outside of the boundary layer detached at the orifice (see Fig. 9 and explanation given in [55], Fig. 1). The immediate consequence of this particular flow structure is that the transversal pressure gradients are negligible only sufficiently far downstream of the inner lip of the nozzle: In fact, they become negligible at the axial downstream station where the vena contracta effect ends, i.e., where the streamlines become almost parallel. It occurs relatively close to the inner orifice plane, at an approximate $D / 2$ downstream distance. From this point downstream (before shear instabilities of the gas stream with the external environment develop), the gas pressure can be considered almost constant, equal to the outside stagnation value. It is at this point where the liquid jet diameter obtained from the numerical simulation should be compared to the simplest prediction (10).

\section{E. Scaling of the recirculation zone}

For a given gas flow rate $Q_{g}$ and orifice diameter $D=2 R$, the typical gas velocity close to the meniscus surface can be estimated as $V=Q_{g} /\left(\pi R^{2}\right)$. Given the small $\rho=\rho_{g} / \rho_{l}$ values in liquid jets focused by gas, liquid velocities are much smaller than $V$ everywhere. As the liquid approaches the

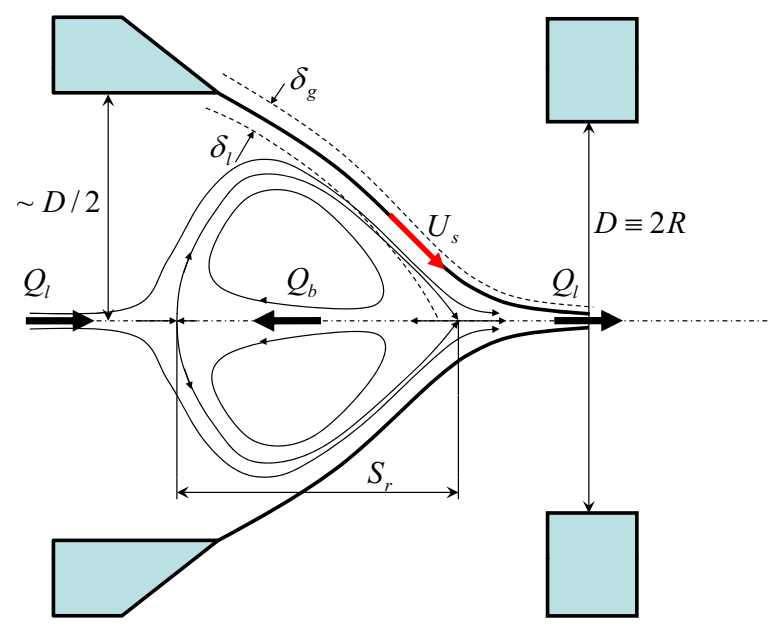

FIG. 22. (Color online) Sketch of the recirculation zone, showing boundary layers, cell size $\left(S_{r}\right)$, and typical velocities.

neck, the boundary layer will collapse (Fig. 22). This implies that at least a liquid flow rate

$$
Q_{r} \sim U_{s} \delta_{l}^{2}
$$

would be drawn into the jet in the absence of recirculation ( $U_{s}$ is the velocity of the interface, that can be obtained from $V$, and the densities and the viscosities ratios [56]). On the contrary, whenever $Q_{l}<Q_{r}$, part of $Q_{r}$ must have been recirculated back into the meniscus (Fig. 22). Therefore $Q_{R}$ can be interpreted as the minimum flow rate for no recirculation (scaled as $Q_{R}=Q_{r} / Q_{g}$ ).

The boundary layer in the liquid meniscus is confined. It grows along the cone during lengths comparable to $R$ (the orifice radius) until the apex of the meniscus is reached. In this area,

$$
\delta_{l} \sim\left(\mu_{l} R / \rho_{l} U_{s}\right)^{1 / 2} .
$$

Whenever there is recirculation, the peripheral boundary layers merge at the meniscus apex and give rise to a jet, whose initial radius at the neck will accordingly be of the same order. In the absence of liquid emission, maximum recirculation will be observed. Experimentally, however, a dripping instability will occur before reaching this limit. In the opposite case (no recirculation), the boundary layers do not merge, and an inviscid core should be observed at the neck. The threshold flow rate for recirculation can therefore be estimated as $Q_{r} \sim U_{s} \delta_{l}^{2}$, a result which happens to be independent of the gas velocity. In effect, by definition of the meniscus boundary layer, the viscous stress $\mu_{l} U_{s} / \delta_{l}^{2}$ must be of the same order as the momentum convection $\rho_{l} U_{s}^{2} / R$, so that, interestingly,

$$
Q_{r} \sim R \frac{\mu_{l}}{\rho_{l}} \Rightarrow Q_{R} \sim \frac{\gamma}{\beta \operatorname{Re}},
$$

where $\beta=\rho_{g} / \rho_{l}$ and $\gamma=\mu_{g} / \mu_{l}$. This scaling is fully confirmed by the numerical simulations: The values of $Q_{\mathrm{R}} \operatorname{Re}$ are 0.6768 for case 1 and 0.6596 for case 2, deviating by less than $2.6 \%$ from the scaling predictions. 
Assume now the recirculation cell to be $S_{r}$ in axial length. The backflow $Q_{b}=\left(Q_{r}-Q_{l}\right)$ will come to rest within a length of the order $S_{r}$. In this length, viscous momentum diffusion should slow down the flow and deflect both the incoming flow injected by the feeding tube and the recirculated flow at the axis (Fig. 22). Thus, viscous and inertia forces should balance within that length $S_{r}$ : In other words, the liquid Reynolds number associated to axial lengths of order $S_{r}$ should be of order unity so as to deflect the unidirectional flow issuing from the feed tube (Hagen-Poiseuille). This is in analogy to the entry length or exit length in laminar pipe flow. Two cases need to be considered, depending of the relative size of the cell compared to the feed tube radius $R_{1}$ :

(i) When $S_{r}<R_{1}$, viscous stress, of the order $O\left(\mu_{l} Q_{b} S_{r}^{-3}\right)$, balances inertia, $O\left(\rho_{l} Q_{b}^{2} S_{r}^{-4}\right)$, which leads to $S_{r} \sim \rho_{l} Q_{b} \mu_{l}^{-1}$.

(ii) When $S_{r}>R_{1}$, viscous stress, $O\left(\mu_{l} Q_{b} R_{1}^{-3}\right)$, balances inertia, $O\left(\rho_{l} Q_{b}^{2} R_{1}^{-3} S_{r}^{-1}\right)$, leading again to $S_{r} \sim \rho_{l} Q_{b} \mu_{l}^{-1}$.

Interestingly enough, again, the length of the recirculation flow is independent of the gas flow for any given geometry. The latter scaling can be expressed in nondimensional terms as

$$
s_{R} \equiv S_{r} / R \sim \boldsymbol{\rho}_{l}\left(Q_{r}-Q_{l}\right) / \boldsymbol{\beta}_{l}^{-1} R^{-1} .
$$

Using Eq. (18), one may write

$$
s_{R}=C_{1}-C_{2} \operatorname{Re}_{R},
$$

where $\operatorname{Re}_{R}=\rho_{l} Q_{l} /\left(\mu_{l} R\right)$ is a Reynolds number of the liquid flow, and $C_{1}$ and $C_{2}$ are constants which depend on the geometry only (i.e., $R_{1} / R, H / R$, etc.). In our case, we have represented all our measured $s_{r}$ values from numerical simulations versus $\operatorname{Re}_{R}$ in Fig. 23. Linear fitting to all points leads to $C_{1}=2.636$ and $C_{2}=0.0819$ with a correlation coefficient of 94.4\%. Equation (22) can be expressed in terms of $Q_{R}-Q$ and $\mathrm{Re}$ as well, as

$$
s_{R}=k \gamma \beta^{-1}\left(Q_{R}-Q\right),
$$

where $k$ is again a constant depending on the geometry only, in full agreement with expression (8), as anticipated by numerical experiments.

\section{EXPERIMENTS}

In the following, we provide experiments corresponding to the same local geometrical parameters as in cases 1 and 2 in the vicinity of the exit orifice. The basic flow focusing chamber is a box consisting of five aluminum faces and one clear methacrylate face. It is $5 \times 5 \times 5.65 \mathrm{~cm}^{3}$, with its longest side along the capillary-orifice axis. The chamber is situated with the methacrylate face horizontal and pointing upwards, the capillary being located parallel to this face. The orifice is made in a stainless steel orifice disk attached to the box side, perpendicular and opposite to the capillary. The disk is $4.0 \mathrm{~mm}$ in diameter with a thickness of $75 \mu \mathrm{m}$ and an orifice of diameter $0.200 \mathrm{~mm}$. Both the air tube and the capillary enter through the face opposite the orifice. After the capillary tube has been aligned with the orifice, the distance $H$ from the tube to the orifice can be simply adjusted by carefully sliding the capillary in its housing on the opposite

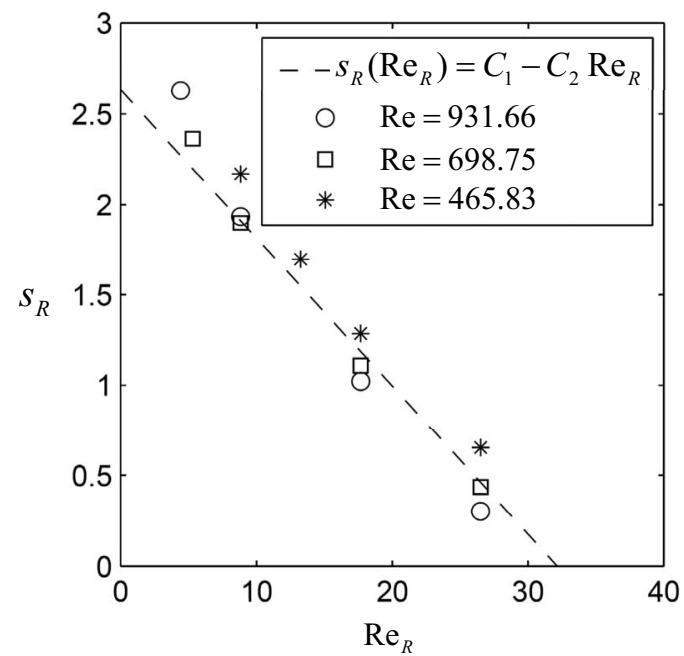

FIG. 23. Recirculation cell size $s_{r}$ as a function of $\mathrm{Re}_{R}$ : Dots, squares, and stars are obtained by numerical simulation; the line is a theoretical prediction resulting from dimensional arguments. An additional series of simulations have been performed for an intermediate gas flow condition $(\mathrm{Re}=698.75$ and $\mathrm{We}=18.31)$ to assess the validity of the scaling proposed: Note the good degree of collapse obtained. The small deviations can be attributable to the small differences in the geometry of the cone for different gas flow conditions.

face to the orifice disk. $H$ is measured with a microscope through the methacrylate face. Figure 24 shows some views of the feeding tube-orifice setup as seen through the thick methacrylate window (inevitable liquid spills leave behind some debris on the inner face of the window causing a blurred image). In particular, Fig. 24(a) shows the geometry numerically simulated in this work.

After setting $H$ and ensuring that the capillary is perfectly coaxial with the nozzle orifice, the pressure is set using a pressure gauge and a pressure meter. A water flow rate is then supplied using a syringe pump (Cole-Palmer $74900 \mathrm{Se}$ ries) with a $20 \mathrm{ml}$ syringe. The system is given sufficient time to relax until either a characteristic steady or unsteady flow is present. This can be checked by illuminating the jet that exits the orifice or by looking at the meniscus when the distance $H$ is $0.100 \mathrm{~mm}$ or greater. Unsteady jet flow appears very faint to the naked eye and contains thin streaks of water along with large scattered spray. This is in significant contrast to steady jet flow, which has bright illumination as a result of a finer, concentrated stream with uniform characteristics. In experiments where the meniscus was visible, it was also possible to discriminate steady versus unsteady flow [see Fig. 24(b), (A) jetting; and (B) dripping], in perfect correlation with the spray observations: A steady meniscus had sharp edges and a clear, unwavering glasslike appearance [Fig. 24, part (A), see steady jet reflected in the metal plate], while an unsteady meniscus had blurred edges and flickered [Fig. 24, part (B), no jet is visible at all]. Both the jet test and meniscus test displayed clear and abrupt transitions between the two states. Once unsteady flow is established for a given pressure, the rate determined by the syringe pump is increased in steps of $0.1 \mathrm{ml} / \mathrm{h}$. After each flow rate increase, a $30 \mathrm{~s}$ waiting period was established, so as to 

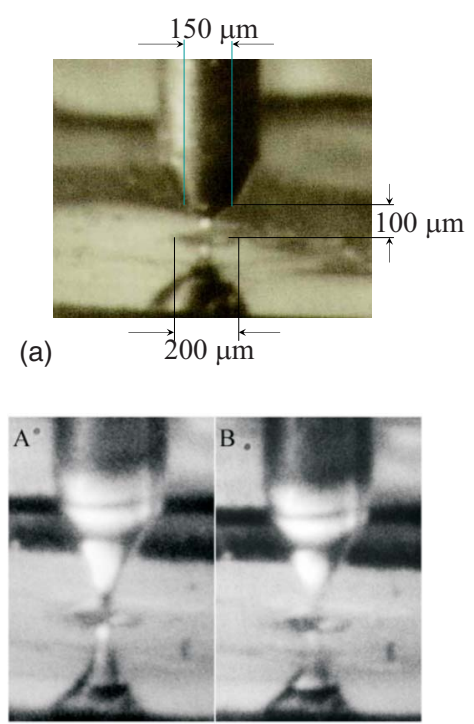

(b)

FIG. 24. (Color online) (a) Experimental tube-orifice setup as numerically simulated in this work $\left(D=200 \mu \mathrm{m}, D_{1}=150 \mu \mathrm{m}, H\right.$ $=100 \mu \mathrm{m}$; here, $\Delta P=10 \mathrm{KPa}, Q_{l}=3 \mathrm{~mL} / \mathrm{h}$ ). (b) Photographs of experimental conditions with two times the distance from the feeding tube to the exit orifice, using a different tube material (fused silica): (A) Jetting $\left(D=200 \mu \mathrm{m}, D_{1}=150 \mu \mathrm{m}, H=200 \mu \mathrm{m}, \Delta P=30 \mathrm{KPa}\right.$, $Q_{l}=6.1 \mathrm{~mL} / \mathrm{h}$ ) and (B) dripping [as in (A) with $Q_{l}=2.8 \mathrm{~mL} / \mathrm{h}$ ].

ensure that the system had relaxed and all the readings were accurate. This period has been chosen after it was found that $15 \mathrm{~s}$ was not enough to observe fluctuations in the system: occasionally a steady regime would revert back to an unsteady one after the $15 \mathrm{~s}$ period. The $30 \mathrm{~s}$ delay has proven long enough to accurately characterize the flow. Accordingly, the rate was increased until the unsteady jet sharply transitioned to a steady one; at this point, the flow rate was read from the syringe pump and recorded as the minimal flow rate (increasing, or up; Fig. 25). Keeping this same steady flow rate the process is then reversed to find the minimum decreasing (or down) flow rate (steps of $0.1 \mathrm{ml} / \mathrm{h}$ and intervals of $30 \mathrm{~s}$ until an unsteady regime developed). When the flow became unsteady, a rate $0.1 \mathrm{ml} / \mathrm{h}$ above the reading on the syringe pump was recorded, since the rate which produced the last steady flow (i.e., minimum flow rate) was one step $(0.1 \mathrm{ml} / \mathrm{h})$ higher. The resulting value was recorded as the minimal (decreasing, or down) flow rate. This process is repeated for varying pressures and distances of $H$ to get an accurate mapping of minimal jetting flow rates as a function of varying geometry and flow conditions. Following this procedure, we collected the experimental data plotted in Fig. 25 for $H / R=1$. The gas (air) pressure $\Delta P$ increases as indicated by the arrow.

Six conditions numerically tested for cases 1 and 2 are plotted in Fig. 25. In order to make our results readily translatable in most of the capillary jet stability literature (which uses the jet radius as a characteristic length), we may introduce liquid Reynolds and Weber numbers consistent with previous definitions and using scaling law (10),

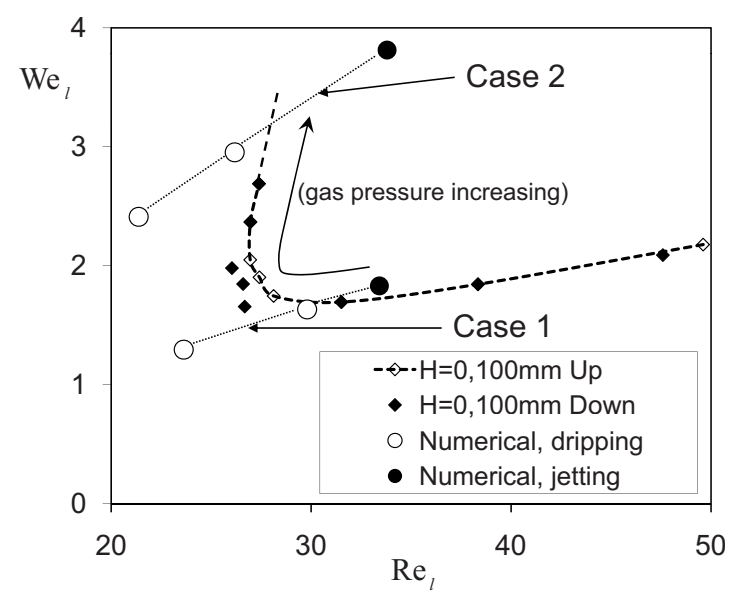

FIG. 25. Jetting-dripping transition in the $\left\{\mathrm{Re}_{l}, \mathrm{We}_{l}\right\}$ plane. Diamonds: Experimentally determined conditions. Filled symbols, liquid flow rate decreasing, "down;" open symbols, liquid flow rate increasing, "up." In most cases, both "up" and "down" points coincide. Circles, numerically tested conditions. Filled circles, jetting conditions. Open circles, dripping conditions.

$\operatorname{Re}_{l}=\left(\frac{2}{\pi^{2}}\right)^{1 / 4}\left(\frac{\rho_{l}^{3} Q_{l}^{2} \Delta P}{\mu_{l}^{4}}\right)^{1 / 4}, \quad \mathrm{We}_{l}=\left(\frac{8}{\pi^{2}}\right)^{1 / 4}\left(\frac{\rho_{l} Q_{l}^{2} \Delta P^{3}}{\sigma^{4}}\right)^{1 / 4}$

As it follows from the plot, using these definitions, jetting or dripping conditions are accurately predicted by the numerical model. This lends additional support to the use of full VOF simulation analysis in flow focusing systems.

\section{CONCLUSIONS}

The cone-jet geometry associated with flow focusing has been handled by a diversity of tools, numerical, experimental, and theoretical. Order-of-magnitude estimations follow from dimensional arguments: Such procedures contribute a valuable theoretical framing and provide the scaling criteria for data representation. Analytical approaches are generally based on the consideration of a perfectly cylindrical infinite jet, a simplification that ignores the influence of the meniscus (a source of instability) and the role of streamline convergence or divergence in the jet. Experiments are burdened by the diversity of influencing parameters and visualization difficulties associated with the small scale of the meniscus and jet.

In this paper, experimental results are backed up by a numerical simulation based on VOF elements. Numerical schemes allow a more systematic exploration of the parametric influence. In addition, the shortcomings of theoretical models (unavoidable in a situation where the geometry of the fluid domain is complex, as in a cone-jet flow pattern) are overcome, and a detailed description of the streamlines can be readily obtained.

The key results of the above exploration are the following:

(i) The theoretical scaling leading to jet diameter estimates is confirmed by the simulation. The expressions for 
flow focusing scales, notwithstanding their simplicity, are therefore to be considered a reliable shortcut for the prediction of jet dimensions.

(ii) The complete sequence from meniscus growth to jet emission (jetting regime) and to the sequential filling of drops (dripping regime) is portrayed in detail.

(iii) The jetting-dripping transition is documented in detail, both by experiment and simulation. A two-branch structure is observed in the plot, showing the simultaneous influence of the jet and the meniscus as instability sources. Incipient dripping (Fig. 13) is shown to give rise to highly irregular fluctuations; while fully developed dripping (Fig. 15) produces perfect cycles of drop detachment.

(iv) A recirculation cell is identified in the jetting regime at the meniscus tip. This occurrence appears to be linked to intensive forcing by the gas sheath, leading to high interface velocity along the meridians; the issuing jet is unable to convey all of the mobilized flow, so that a return flow around the axis is observed. The recirculation cell grows as the liquid flow rate is reduced: Eventually, dripping conditions are reached. Similar recirculation cells have been observed in electrospray cones, under thread emission, and in liquidliquid two-dimensional flow focusing, assisted or not by a surfactant [5,29,31]. All of the recirculation instances reported thus far appear to share a common attribute: Strong interfacial forcing, either electric, capillary or hydrodynamic. (v) A reliable scaling is provided, identifying the parametric conditions where recirculation is to be expected and estimating the size and flow rates of the cell.

A key feature in the flow pattern explored is the recirculation cell, and its conceptual link to the merging of the boundary layers which grow from the meniscus edge and fuse together at the neck of the jet. Controllable recirculation is an extremely attractive feature, providing adjustable residence times within a very simple flow setup. The cell can be viewed as a flow trap or reactor, where biosynthesis or chemical operations take place in a protected environment; the liquid flow rate can be increased to flush the recirculation products.

An additional focus deals with the peculiarities of the jetting and dripping regimes under the influence of a coflowing sheath current. The aspect of the jet and droplet train and the dynamics of the meniscus (an indicator of dripping) are a contribution to a problem whose complexity forbids a global theoretical approach.

\section{ACKNOWLEDGMENTS}

This work has been supported by the Spanish Ministry of Science and Education, Contract No. DPI2004-07197, and partially supported by European Commission through Grant No. COOP-CT-2005-017725. Thorough discussions of one of the authors (A.M.G.C.) with Dr. Joan Rosell-Llompart are warmly acknowledged.
[1] O. A. Basaran, AIChE J. 48, 1842 (2002).

[2] H. Song, D. L. Chen, and R. F. Ismagilov, Angew. Chem., Int. Ed. 45, 7336 (2006).

[3] A. D. Griffiths and D. S. Tawfik, Trends Biotechnol. 24, 395 (2006).

[4] G. I. Taylor, Proc. R. Soc. London, Ser. A 146, 501 (1934).

[5] R. Suryo and O. A. Basaran, Phys. Fluids 18, 082102 (2006).

[6] W. W. Zhang, Phys. Rev. Lett. 93, 184502 (2004).

[7] R. J. Haywood, M. Renksizbulut, and G. D. Raithby, AIChE J. 37, 1305 (1991).

[8] R. T. Collins, J. J. Jones, M. T. Harris, and O. A. Basaran, Nat. Phys. 4, 149 (2007).

[9] E. Lac and G. M. Homsy, J. Fluid Mech. 590, 239 (2007).

[10] H. N. Oguz and A. Prosperetti, J. Fluid Mech. 257, 111 (1993).

[11] A. M. Gañán-Calvo, Phys. Rev. Lett. 80, 285 (1998).

[12] A. M. Gañán-Calvo and J. M. Gordillo, Phys. Rev. Lett. 87, 274501 (2001).

[13] S. Takeuchi, P. Garstecki, D. B. Weibel, and G. M. Whitesides, Adv. Mater. (Weinheim, Ger.) 17, 1067 (2005).

[14] S. L. Anna, N. Bontoux, and H. Stone, Appl. Phys. Lett. 82, 364 (2003).

[15] P. Garstecki, I. Gitlin, W. DiLuzio, E. Kumacheva, H. A. Stone, and G. M. Whitesides, Appl. Phys. Lett. 85, 2649 (2004).

[16] M. Seo, Z. H. Nie, S. Q. Xu, M. Mok, P. C. Lewis, R. Graham, and E. Kumacheva, Langmuir 21, 11614 (2005).

[17] M. J. Jensen, H. A. Stone, and H. Bruus, Phys. Fluids 18,
077103 (2006).

[18] A. S. Utada, E. Lorenceau, D. R. Link, P. D. Kaplan, H. A. Stone, and D. A. Weitz, Science 308, 537 (2005).

[19] C. Berkland, E. Pollauf, D. W. Packa, and K. Kim, J. Controlled Release 96, 101 (2004).

[20] L. Martín-Banderas, A. Rodríguez-Gil, A. Cebolla, S. Chávez, T. Berdún-Alvarez, J. M. Fernandez-Garcia, M. FloresMosquera, and A. M. Gañán-Calvo, Small 1, 688 (2005).

[21] L. Martín-Banderas, M. Flores-Mosquera, P. Riesco-Chueca, A. Rodríguez-Gil, A. Cebolla, S. Chávez, and A. M. GañánCalvo, Adv. Mater. (Weinheim, Ger.) 18, 559 (2006).

[22] A. M. Gañán-Calvo and P. Riesco-Chueca, J. Fluid Mech. 553, 75 (2006).

[23] A. M. Gañán-Calvo, M. A. Herrada, and P. Garstecki, Phys. Rev. Lett. 96, 124504 (2006).

[24] A. M. Gañán-Calvo, Phys. Rev. E 75, 027301 (2007).

[25] D. Erickson, Microfluid. Nanofluid. 1, 301 (2005).

[26] V. Cristini and Y.-Ch. Tan, Lab Chip 4, 257 (2004).

[27] M. M. Dupin, I. Halliday, and C. M. Care, Phys. Rev. E 73, 055701 (2006).

[28] M. W. Weber and R. Shandas, Microfluid. Nanofluid. 3, 195 (2007).

[29] A. Barrero, A. M. Gañán-Calvo, J. Dávila, A. Palacio, and E. Gómez-González, Phys. Rev. E 58, 7309 (1998).

[30] M. A. Herrada and A. Barrero, Phys. Rev. E 66, 036311 (2002).

[31] S. L. Anna and H. C. Mayer, Phys. Fluids 18, 121512 (2006).

[32] J. M. Fernandez and G. M. Homsy, Phys. Fluids 16, 2548 
(2004).

[33] R. Krechetnikov and G. M. Homsy, Phys. Fluids 16, 2556 (2004).

[34] D. B. Kothe and W. J. Rider, Fluid Dyn. 3, 19 (1995).

[35] S. O. Unverdi and G. Tryggvason, J. Comput. Phys. 100, 25 (1992).

[36] D. J. E. Harvie and D. F. Fletcher, J. Comput. Phys. 162, 1 (2000).

[37] E. Aulisa, S. Manservisi, and R. Scardovelli, J. Comput. Phys. 188, 611 (2003).

[38] S. Tanguy and A. Berlemont, Int. J. Multiphase Flow 31, 1015 (2005).

[39] C. W. Hirt and B. D. Nichols, J. Comput. Phys. 39, 201 (1984).

[40] P. Heinrich, Int. J. Eng. Fluid Mech. 4, 403 (1991).

[41] A. Tomiyama, A. Sou, H. Minagawa, and T. Sakaguchi, JSME Int. J., Ser. B 36, 51 (1993).

[42] B. Lafaurie, C. Nardone, R. Scardovelli, S. Zaleski, and G. Zanetti, J. Comput. Phys. 113, 134 (1994).

[43] B. Van Leer, J. Comput. Phys. 32, 101 (1979).

[44] O. Ubbink, Ph.D thesis, Imperial College of Science, Technology and Medicine, London 1997.
[45] R. I. Issa, J. Comput. Phys. 62, 40 (1986)

[46] R. Shaw, The Dripping Faucet as a Model Chaotic System (Aerial, Santa Cruz, 1984).

[47] N. Fuchikami, S. Ishioka, and K. Kiyono, J. Phys. Soc. Jpn. 68, 1185 (1999).

[48] B. Ambravaneswaran, S. D. Phillips, and O. A. Basaran, Phys. Rev. Lett. 85, 5332 (2000).

[49] B. Ambravaneswaran, H. J. Subramani, S. D. Phillips, and O. A. Basaran, Phys. Rev. Lett. 93, 034501 (2004).

[50] H. J. Subramani, H. K. Yeoh, R. Suryo, Q. Xu, B. Ambravaneswaran, and O. A. Basaran, Phys. Fluids 18, 032106 (2006).

[51] P. Coullet, L. Mahadevan, and C. S. Riera, J. Fluid Mech. 526, 1 (2005).

[52] E. Villermaux, P. Marmottant, and J. Duplat, Phys. Rev. Lett. 92, 074501 (2004).

[53] R. L. Sani and P. M. Gresho, Int. J. Numer. Methods Fluids 18, 983 (1994).

[54] P. M. Morse and H. Feshback, Methods of Theoretical Physics (McGraw-Hill, New York, 1953).

[55] A. M. Gañán-Calvo, Phys. Rev. E 78, 026304 (2008).

[56] A. M. Gañán-Calvo, Phys. Rev. Lett. 98, 134503 (2007). 